\title{
由 由
}

UNIVERSIDAD

DEL PACÍFICO

FACULTAD DE CIENCIAS

EMPRESARIALES

\section{Un acercamiento al deporte y al fútbol como negocio y al funcionamiento, problemática y mejora del fútbol peruano}

\author{
Emilio Humberto García Vega \\ MBA, Universidad del Pacífico. Licenciado en Administración, \\ Universidad del Pacífico. Asesor, Consultor, Profesor e \\ Investigador en Estrategia Empresarial. Especialista en \\ Planeamiento, Implementación y Control de estrategias \\ empresariales y de marketing. Autor de los libros ¿Cómo \\ generar valor en las empresas? (2012) y ¿Qué hace especiales \\ a las empresas?: La Ventaja Competitiva a inicios del Siglo \\ XXI (2011), entre otros.
}

\section{Resumen}

El presente artículo trata de aproximarse al deporte, más específicamente al fútbol, como negocio y acercarse a su realidad, funcionamiento y problemática en el Perú. En esta línea, en primer lugar se desarrolla un marco teórico general que permite conocer cómo se presenta la transformación del deporte y del fútbol hacia un modelo de negocio rentable, conocer brevemente cómo se da la generación de valor de una institución deportiva; y además, se presentan los factores críticos del éxito de los clubes con mayores ingresos en el mundo. En segundo lugar, se presenta un acercamiento al fútbol peruano desde lo deportivo, legal y económico además de aproximarse a su funcionamiento mediante la explicación de la forma en que se desarrolla el torneo profesional de fútbol peruano, la manera cómo funciona el fútbol amateur de dicho país, además de cómo se maneja institucionalmente este deporte en esta nación, entre otros aspectos. En tercer lugar, se presentan los problemas del fútbol profesional de dicho país sobre la base de fuentes secundarias y de quince entrevistas desarrolladas a periodistas especializados y dirigentes. Finalmente, se cierra este trabajo con algunas conclusiones y recomendaciones enfocadas a la mejora del fútbol peruano.

\section{Palabras clave:}

Gestión y administración deportiva, gestión y administración del fútbol, fútbol peruano, estrategia. 


\section{Objetivos}

\section{Objetivos principales}

- Desarrollar una aproximación al deporte y más específicamente al fútbol como negocio.

- Desplegar un acercamiento a la realidad, el funcionamiento y la problemática del fútbol peruano.

\section{Objetivos secundarios}

- Desarrollar un breve marco teórico general que permita conocer cómo se presenta la transformación del deporte y del fútbol hacia un modelo de negocio rentable.

- Conocer brevemente cómo se da la generación de valor de una institución deportiva.

- Presentar los factores críticos del éxito de los clubes con mayores ingresos en el mundo.

- Desarrollar un acercamiento al fútbol peruano desde lo deportivo, legal y económico.

- Aproximarse al funcionamiento del fútbol peruano mediante la explicación de la forma en que se desarrolla su torneo profesional de fútbol, la manera cómo funciona su fútbol amateur, además de cómo se maneja institucionalmente este deporte en dicha nación.

- Presentar los problemas del fútbol profesional de dicho país sobre la base de fuentes secundarias y de quince entrevistas desarrolladas a periodistas especializados y dirigentes.

- Presentar algunas conclusiones y recomendaciones enfocadas a la mejora del fútbol peruano.

\section{Metodología}

- En primer lugar, se presentará una introducción a manera de presentación del trabajo desarrollado.

- En segundo lugar, se desarrolla una revisión bibliográfica del tema del deporte como negocio y más específicamente del fútbol como negocio. Además, de este recorrido sobre desarrollos teóricos, se revisan los factores críticos del éxito de los principales clubes de fútbol profesional del mundo. Sobre la base de esta revisión, se plantea un marco teórico que sustenta el trabajo.

- En tercer lugar se revisan fuentes secundarias de diverso tipo para aproximarse al funcionamiento, realidad y problemática del fútbol en el Perú.

- En cuarto lugar, se desarrollaron quince entrevistas a periodistas especializados y dirigentes del fútbol peruano para ahondar en la problemática del fútbol de dicho país.

- Finalmente, sobre la base del trabajo desarrollado, se procede a formular conclusiones y recomendaciones enfocadas a la mejora del fútbol peruano.

\section{INTRODUCCIÓN}

En el Perú, a pesar de no clasificar a un mundial de fútbol de mayores desde el año de 1982, el fútbol siempre genera grandes expectativas y fanatismos. La inesperada y exitosa campaña deportiva de la selección nacional de mayores de dicho deporte en la Copa América de 2011 desarrollada en Argentina, hizo que el Perú se paralizara cuando la selección jugaba y más aún, cuando fue avanzando inusitadamente en el mencionado torneo, llegando a un tercer lugar.

Sin embargo, el éxito en cuanto a fanatismo, expectativa, preferencia y simpatía, no se refleja en el éxito comercial y económico de los clubes de fútbol peruanos. Esto es muy común en los países de la región, siendo constante la difusión de noticias acerca de la insolvencia, las deudas $y$, en suma, de los problemas económicos de las instituciones deportivas de fútbol. Sin embargo, cabe mencionar que los clubes de cada país son acogidos por entes rectores y organizadores cuyas gestiones muchas veces no han potenciado que el fútbol sea una opción de negocio rentable más allá de las selecciones de cada país que presentan ingresos propios basados fundamentalmente en 
los derechos televisivos, venta de publicidad y comercialización de entradas.

Esta complicada situación futbolística de la mayoría de los países de la región, dista diametralmente del principal mercado de este deporte, el europeo. En esta línea, en un informe de la firma internacional Deloitte, titulado Conclusiones y un análisis desde la perspectiva Latinoamericana (Deloitte 2011), se afirma que en los últimos años el fútbol ha sufrido muchos cambios y Latinoamérica no ha seguido el mismo ritmo de Europa. Los veinte principales clubes del viejo continente pasaron de facturar $€ 2.1$ billones en la temporada 1996-1997 a € 4.3 billones en la temporada 2009-2010.

En este informe, se afirma que en Latinoamérica no pueden identificarse factores de éxito en los clubes profesionales de fútbol con certeza, dado que no existe información confiable. El único dato que se tiene es que la venta de jugadores representa el mayor porcentaje de ingresos para los clubes de la región latinoamericana. Así, por ejemplo, representa un 50\% en Argentina, 30\% en Brasil y $23 \%$ en México. La exportación de jugadores hace que el producto deportivo de los campeonatos latinoamericanos se empobrezca ya que los mejores jugadores emigran y se quedan en el medio local aquellos de nivel inferior, lo que hace que el nivel de los campeonatos en Latinoamérica se encuentre muy por debajo de su potencial. Deloitte plantea que para empezar a tener éxito en Latinoamérica, debe empezarse por hacer buenas gestiones en las organizaciones relacionados con el fútbol, para lo cual debe apuntarse aun mayor profesionalismo en las dirigencias de la región.

Este trabajo pretende presentar un acercamiento a la realidad del fútbol peruano profesional partiendo de un ámbito general y desembarcando en el caso específico de un club peruano. Así, en primer lugar se desarrolla un marco teórico general que permite conocer cómo se presenta la transformación del fútbol hacia un modelo de negocio rentable, conocer brevemente cómo se da la generación de valor de una institución deportiva; y además, se presentan los factores críticos del éxito de los clubes con mayores ingresos en el mundo
En segundo lugar, se presenta un acercamiento al fútbol peruano desde lo deportivo, legal y económico además de aproximarse a su funcionamiento profesional y amateur. En tercer lugar, se presentan los problemas del fútbol profesional de dicho país; luego —en cuarto lugar-, se cuenta la experiencias del Club Universidad San Martín de Porres. Finalmente, se cierra este trabajo con algunas conclusiones y recomendaciones.

\section{Marco teórico: El deporte y el fútbol como negocio; y la gestión eficiente de los clubes de fútbol con mayores ingresos en el mundo}

En este acápite se desarrollará una revisión bibliográfica que permitirá una aproximación a cómo el deporte se transformó en un modelo empresarial convirtiéndose en una atípica empresa de espectáculo y entretenimiento. Seguidamente, se explica brevemente cómo los clubes de fútbol siguen esta tendencia y también, se convierten en modelos de negocio rentables. Luego, se establece un acercamiento hacia el valor de una institución deportiva para, hacia el final de esta revisión; presentar brevemente los elementos que han hecho posible la generación de ingresos de los dos clubes de fútbol con mayor facturación en el mundo.

\section{IV.1 El deporte como una atípica empresa de espectáculo y entretenimiento}

Altuve (2011) plantea que el deporte como atípica empresa transnacional de espectáculo y entretenimiento aparece a partir del año 1982 cuando se presentan los siguientes hechos: i) Se dispone de una moderna empresa de marketing o patrocinio deportivo, capaz de comercializar los más importantes eventos deportivos del mundo; ii) Se logra la centralización de los derechos de comercialización de los Juegos Olímpicos (JO) en el Comité Olímpico Internacional (COI) y la concentración del patrocinio deportivo mundial en ISL; y iii) Se profundiza la profesionalización del deportista-competidor y se tiende a la eliminación del atleta aficionado o amateur.

Este autor afirma que la función del modelo deportivo globalizador transnacional (MDGT) es 
producir un espectáculo rentable. Así, el MDGT se inició en la década de los ochenta y principios de los noventa y afirma que constituye una tendencia irreversible. Horst Dassler crea ISL en 1982, con esta empresa logra comercializar dos de los eventos más importantes del mundo: el Mundial de Fútbol y los Campeonatos Mundiales de Atletismo. Esto lo logró asegurando los derechos de comercialización del fútbol internacional, promoviendo el regreso de Coca Cola al patrocinio del fútbol y distribuyendo exclusivos e interesantes paquetes de comunicaciones a grandes transnacionales del mundo. Luego, la incorporación de los Juegos Olímpicos al proceso de comercialización centralizado del fútbol, atletismo y otras disciplinas deportivas significó la instalación del nuevo modelo deportivo mercantil mundial. Esta incorporación implicó: a) la negociación de Horst Dassler (Gerente General y dueño de Adidas) con los Comités Olímpicos de mayor poder económico (EEUU, Alemania Occidental, Inglaterra, Francia, Japón y Australia) para consolidar la comercialización centralizada; y b) Recaudar entre nueve compañías transnacionales (Coca-Cola, Visa, Brother, Federal Express, 3M, Time-Life, National Panasonic, Kodak, y Philips) más de 100 millones de dólares para el primer Programa Olímpico de los Juegos Olímpicos de Seúl 1988 a cambio de obtener la exclusividad en la utilización de los símbolos olímpicos en cualquier parte del mundo.

ISL es la empresa de marketing o patrocinio modelo de la globalización en el deporte. Es el instrumento moderno que resume la comercialización deportiva, es la organización que promociona, impulsa y organiza proyectos de desarrollo del deporte como un espectáculo, lo suficientemente atractivos para que sean financiados por grandes empresas patrocinadoras que adquieren el derecho de utilización exclusiva de la marca, símbolos y logos en los eventos deportivos que patrocinan. Las grandes sumas de dinero pagadas al COI por parte del Comité Olímpico Nacional (CON) de EEUU y la capacidad de consumo público de EEUU consolidó el modelo. Por el lado de los atletas, se produjo su profesionalización de manera que en los países desarrollados, casi se han eliminado la figura del atleta amateur.

Cabe mencionar que la transnacional Adidas desempeñó un papel protagónico en la constitución de la empresa deportiva transnacional de espectáculo y entretenimiento porque fue una de las principales responsables de la creación de la moderna empresa de marketing o patrocinio de marketing o patrocinio deportivo. Articuló o integró su experiencia en la promoción de sus artículos por parte de los atletas y por los árbitros-jueces en los eventos deportivos. Así mismo, entendió el nuevo papel de la televisión en el deporte, creó las primeras empresas de marketing o patrocinio deportivo y contribuyó a la conversión de grandes empresas transnacionales en patrocinadores deportivos. Así, Adidas se convirtió en un gran «sponsor» del deporte. Además, creo ISL, la empresa de patrocinio o «marketing deportivo» modelo en la globalización que llevó a cabo el programa de comercialización de los principales eventos deportivos mundiales.

Adidas invirtió esfuerzos y recursos en el ascenso al poder, en la organización deportiva mundial; de una nueva generación de dirigentes partidarios de la comercialización deportiva, con quienes estableció una amplia y estrecha relación de colaboración para impulsar la comercialización del deporte mundialmente. En esta línea estuvieron, Joao Havelange (presidente de la Federación Internacional de Fútbol Asociado (FIFA) desde 1974); Juan Antonio Samaranch (presidente del COI, desde 1980); Primo Nebiolo (presidente de la FIAA, desde 1981); Robert Hemlick (presidente del CON de EEUU y miembro del COI en 1985, reelecto como presidente del CON de EEUU y ascendido a miembro de la Junta Directiva del COI en 1989). La transnacional alemana se impuso como la marca del deporte al ser identificada como símbolo e ícono del mismo. Esto lo logró al convertirse en el proveedor oficial de artículos e implementos deportivos de importantes federaciones nacionales e internacionales, utilizados en los más importantes eventos deportivos mundiales, los cuales tenían también el patrocinio de la empresa, además, de estar presente en diversos y amplios escenarios 
asociados o vinculados con la actividad deportiva. Adidas redimensionó el negocio de los artículos e implementos deportivos cuando incursionó protagónicamente en la comercialización del deporte y se posicionó como una de las principales empresas mundiales del ramo al tener una intensiva y extensiva promoción y mercadeo de sus productos por todo el mundo.

En suma, la participación de Adidas fue fundamental en la consolidación definitiva de un nuevo patrón de comercialización deportiva adaptado y demandado por las nuevas exigencias económicas, políticas, sociales, tecnológicas y culturales. En definitiva, fue el motor de un nuevo modelo deportivo mundial: la globalización o transnacionalización deportiva. Esta compañía fue uno de los creadores más importantes de la estructura mundial de negocios en el deporte, en un proceso donde ella misma como empresa de artículos e implementos deportivos y la compañía de comercialización ISL, se convirtieron en dos de las instituciones más poderosas e influyentes del mundo en materia deportiva

Así, hacia fines del siglo XX se configuró la fisonomía de la empresa deportiva transnacional atípica de espectáculo y entretenimiento que tiene las siguientes características:

- Su objetivo fundamental es producir un espectáculo crecientemente rentable.

- Su radio de acción es el mundo entero, ya que está presente en todos los países.

- Es totalmente monopólica, no ofrece simultáneamente dos o más versiones del mismo espectáculo.

- Está constituida por unidades socioeconómicas que, por una parte, son autónomas e independientes en su dinámica y funcionamiento, y por otra, son interdependientes porque dependen unas de las otras para garantizar el éxito del ciclo productivo deportivo. Dichas unidades son: organizadores del espectáculo (una organización deportiva mundial (ODM), el gobierno del deporte; clubes profesionales; transnacionales avaladas por la ODM; y transnacionales independientes de la ODM); transnacionales patrocinadoras $\mathrm{o}$ «sponsors»; transnacionales de medios de comunicación; estados nacionales; deportistas- competidores; y públicoconsumidor.

- Todas las unidades socio-económicas con la excepción del público-consumidor, los deportistas-competidores no exitosos y los estados nacionales no exitosos en términos deportivos, disponendedivisiones, secciones o empresas de mercadeo o marketing.

- Utiliza la ciencia y la tecnología más avanzada del mundo en todas las dimensiones (organizativo; comunicacional; en los artículos e implementos usados en las competencias; en todo el proceso de competencias) del espectáculo deportivo.

La empresa deportiva transnacional atípica de espectáculo y entretenimiento adelanta la creación y desarrollo de estructuras de mercadeo y comercialización propias de la economía globalizada con:

- La creación de nuevos eventos deportivos de carácter mundial (campeonato mundial de clubes de fútbol, copa mundial de béisbol, entre otros) y la ampliación de algunos ya existentes con el objetivo de aumentar su cobertura e influencia.

- Campañasdiversasrealizadas porlosgrandes clubes profesionales transnacionales (juegos de exhibición, de pretemporada, giras profesionales de los equipos $\mathrm{y} / \mathrm{o}$ jugadores) destinadas a ampliar su radio de acción e influencia en el mundo entero.

- Establecimiento de pactos comerciales y de mercadeo entre grandes clubes profesionales transnacionales de dos disciplinas deportivas diferentes e importantes y procedentes de países de dos continentes distintos. Un ejemplo de esto es la alianza entre el club de fútbol inglés Manchester United y el equipo de béisbol los Yankees de Nueva York.

Según Dávila (2007), el deporte es un negocio muy lucrativo, lo cual es demostrado por las ligas deportivas estadounidenses de fútbol americano (NFL), baloncesto (NBA) y béisbol (MLB); desde hace varios años. Así, la NFL es la liga más rentable 
del mundo con retornos superiores al $15 \%$ anual. Dávila afirma que la gestión del deporte profesional se encuentra en una época de cambios radicales y, como consecuencia, cada vez resulta más compleja. La globalización de la demanda es un hecho, de manera que los equipos europeos venden más camisetas internacionalmente que en su propio país y los partidos, por ejemplo, de la liga inglesa se siguen en más de 150 países. Estas son evidencias que se trata de un mercado que cuenta con un gran potencial.

\section{IV.2 La transformación del fútbol hacia un modelo de negocio rentable}

En la transformación descrita líneas arriba, el fútbol se convierte en un modelo de negocio rentable. Algunos clubes europeos, empezaron a transformar sus formas de gestión. Esta transformación coincide con el cambio de la década de los ochenta a los noventa. En esta línea, según Breitbarth y Harris (2008), las fuentes de ingresos de los clubes de fútbol son: el equipo de fútbol propiamente dicho, las propiedades o edificios con los que cuentan los clubes y, finalmente, sus marcas. En esta línea, el cambio de las fuentes de ingresos de los clubes, se puede resumir en el siguiente cuadro.

\begin{tabular}{|c|c|c|}
\hline \multicolumn{3}{|c|}{$\begin{array}{c}\text { Cuadro } 1 \\
\text { Evolución de las fuentes o ingresos de los clubes de fútbol } \\
\text { (antes y desde la década de los noventa) }\end{array}$} \\
\hline $\begin{array}{l}\text { FUENTES / } \\
\text { INGRESOS }\end{array}$ & $\begin{array}{c}\text { CLUBES } \\
\text { ANTES DE } 1990\end{array}$ & $\begin{array}{c}\text { CLUBES } \\
\text { DESDE } 1990\end{array}$ \\
\hline Equipo de fútbol & $\begin{array}{l}\text { - Ventas de entradas y donaciones } \\
\text { - Sponsorship y publicidad } \\
\text { - Derechos de transmisión (a muy baja escala) }\end{array}$ & $\begin{array}{l}\text { - Venta de entradas y merchandising } \\
\text { - Desarrollo y venta de jugadores } \\
\text { - Sponsorship y publicidad } \\
\text { - Derechos de transmisión } \\
\text { - Otras inversiones secundarias }\end{array}$ \\
\hline Propiedades / Edificios & - Instalaciones de propiedad pública & $\begin{array}{l}\text { - Instalaciones multifuncionales, especialmente } \\
\text { estadios } \\
\text { - Variedad de servicios }\end{array}$ \\
\hline Marcas & - Cargos por membresía al club & $\begin{array}{l}\text { - Promover productos y servicios no relacionados al } \\
\text { fútbol (tarjetas de crédito, seguros, servicio de } \\
\text { telefonía, apuestas) }\end{array}$ \\
\hline
\end{tabular}

Fuente: Breitbarth y Harris (2008).

En los inicios del nuevo siglo, Dobson y Goddard (2001) plantean que se puede distinguir dos grandes categorías de negocios en el fútbol: los negocios tradicionales y la explotación de negocios relacionados con la marca. Los negocios tradicionales se refieren a la explotación del evento deportivo en vivo, lo que se enfoca principalmente a la gestión del aforo del estadio, los aficionados y los pacos VIP, y la venta de derechos de transmisión de los partidos, Cabe mencionar que la explotación de los negocios relacionados con la marca han multiplicado y diversificado los ingresos de los clubes. Seguidamente se presenta un cuadro resumen con las categorías de negocios de los clubes de fútbol. 
Cuadro 2

Categorías de negocios de los clubes fútbol

\begin{tabular}{|c|c|}
\hline MEJORA EN LA GESTIÓN DE NEGOCIOS TRADICIONALES & NEGOCIOS RELACIONADOS CON LA EXPLOTACIÓN DE LA MARCA \\
\hline $\begin{array}{l}\text { 1.Aforo del estadio } \\
\text { 1.1Explotación comercial de las instalaciones } \\
\text { IDirigidos a aficionados } \\
\text { IDirigidos a empresas: } \\
\text { •Venta de entradas (cesión abono) } \\
\text { • Bares del estadio y pabellón } \\
\text { •Exposición trofeo y tour estadio } \\
\text { - Apertura de negocios restauración estadio } \\
\text { •Explotación palcos VIP } \\
\text { • Organización eventos } \\
\text { 1.2Explotación del evento deportivo en vivo } \\
\text { I[Soportes publicitarios estadio } \\
\text { gIPaquete turístico VIP completo } \\
\text { TIEntrada aficionados no abonados } \\
\text { 2. Explotación de la televisión. } \\
\text { 2.1Reparto derechos retransmisión } \\
\text { 2.2Explotación comercial de canales de televisión propios. }\end{array}$ & $\begin{array}{l}\text { 1.Derechos de imagen de futbolistas del club. } \\
\text { Obtención en la explotación de la imagen de los futbolistas. } \\
\text { Negociación de contratos en mercado publicitario). } \\
\text { 2.Acuerdos internacionales de desarrollo de marca. } \\
\text { Búsqueda de socios locales para explotación de la marca del club. } \\
\text { 3.Esquema de patrocinios (sponsorship, partnership) } \\
\text { Contrato de patrocinio principal y paquetes de patrocinios. } \\
\text { 4.Desarrollo de nuevas tecnologías. } \\
\text { Servicios y contenidos en internet. } \\
\text { 5.Desarrollo de programas para socios. } \\
\text { Servicios de carnets con ventajas especiales para los socios. } \\
\text { 6.Programa de licencias (merchandising y licensing). } \\
\text { 7.Retail \& Distribution } \\
\text { Tiendas oficiales y franquicias de las mismas. }\end{array}$ \\
\hline
\end{tabular}

Elaboración propia sobre la base de Dobson y Godard (2001), y Blanco y Forcadell (2006).

La comercialización del fútbol profesional y su alto perfil público, crea la necesidad de integrar la responsabilidad social al juego. Es importante sostener que las organizaciones de fútbol pueden asumir un rol crítico como agentes de cambio social y que pueden utilizar este rol para de forma paralela, hacer crecer y desarrollar el juego. La estrategia de responsabilidad social en el fútbol promueve principios como la inclusión social, la diversificación y el desarrollo de instituciones en espacios sociales donde otros deportes se han ausentado. El fútbol actúa como un medio para lograr objetivos públicos al establecer al juego como una parte integral del desarrollo de la vida en comunidad y de la construcción de relaciones entre diversos agentes como autoridades locales, grupos de apoyo y los medios de comunicación. Los clubes profesionales de fútbol se han convertido en agentes muy valorados en el desarrollo social y en el despegue de economías regionales y regeneración urbana. Además de crear valor económico y social, existe el potencial de que los clubes usen el poder de sus marcas para promover un mayor involucramiento dentro de la sociedad (Breitbarth y Harris 2008).

\section{IV.3 El valor de una institución deportiva}

Enfocándose en los clubes deportivos, según Gastañaduy (2003), el valor de una institución deportiva estará determinado por los componentes de su propio capital intelectual: conocimientos, información, propiedad intelectual, y experiencia. Pero, más propiamente, el valor de una institución deportiva estará dado por muchos otros factores, entre los que se pueden destacar los siguientes:

- $\mathrm{Su}$ rendimiento o performance en competiciones y obviamente su posición destacada a través del tiempo.

- Su gestión del merchandising con gerencia profesional y con un gran enfoque de marketing estratégico, es decir, en función del mercado.

- $\mathrm{Su}$ infraestructura, con los modernos conceptos de estadio-empresa desarrollados especialmente por poderosas instituciones deportivas en los Estados Unidos.

- La calidad y prestigio de su plantel de jugadores, que, con buen manejo de su imagen profesional y personal, contribuye 
a valorar la institución de manera inconmensurable.

- Sus patrocinadores, que mientras más poderosos sean potenciarán la imagen corporativa y, por ende, el valor patrimonial de la institución deportiva. Ello debido a que un patrocinador de marca internacional no invertirá en una institución deportiva que no sea también poderosa.

- Su plantel gerencial ya que sin una solvente y profesional conducción empresarial ninguno de los aspectos mencionados funcionará.

Dávila (2007) plantea que el reto que enfrentan las diferentes ligas y cada uno de sus equipos es cómo transformar la gran demanda existente en recursos que les permitan fichar el mejor talento y ofrecer el mejor producto posible. Por otro lado, las grandes cifras de inversión que se mueven y la complejidad del negocio, demandan directivos tan bien o incluso mejor pagados que directivos de empresas en industrias tradicionales. En esta línea, la liga inglesa ha entendido que para ser la mejor del mundo, tiene que verse a sí misma como una industria que, además de establecer las reglas en el campo, tiene la responsabilidad de crearlas al nivel del mercado. Dávila sostiene que se está comenzando a vislumbrar un nuevo modo de gestionar el fútbol profesional, en el que los jugadores y los técnicos continúan siendo uno de los pilares de los equipos, sin embargo, enfatiza que la acumulación de talento deportivo no servirá si no se gestiona adecuadamente la institución.

\section{IV.4 Factores críticos del éxito de los clubes con mayores ingresos en el mundo}

Existen una serie de equipos que han desarrollado un modelo de gestión orientado al mundo empresarial dentro de los parámetros que Dobson y Godard (2001), Breitbarth y Harris (2008), Gastañaduy (2003), entre otros autores plantean. Si bien el nivel de ingresos o facturación no es la única medida para establecer la gestión exitosa de un equipo de fútbol; resulta interesante revisar el informe de la consultora internacional Deloitte, Football Money League, para el año 2011, en el cual se lista a los veinte clubes de fútbol con mayores ingresos en el mundo.

Cuadro 3

Los veinte clubes de fútbol con mayores ingresos en el mundo (en millones de euros)

\begin{tabular}{|c|c|c|}
\hline POSICIÓN & CLUB & FACTURACIÓN MILLONES DE EUROS (2010-2011) \\
\hline 1 & Real Madrid & 479.50 \\
\hline 2 & Barcelona & 450.70 \\
\hline 3 & Manchester United & 367.00 \\
\hline 4 & Bayern Munich & 321.40 \\
\hline 5 & Arsenal & 251.10 \\
\hline 6 & Chelsea & 249.80 \\
\hline 7 & AC Milan & 211.40 \\
\hline 8 & Inter de Milan & 203.30 \\
\hline 9 & Liverpool & 202.40 \\
\hline 10 & Schalke 04 & 181.00 \\
\hline 11 & Tottenhan Hotspur & 169.60 \\
\hline 12 & Manchester City & 153.90 \\
\hline 13 & Juventus & 150.40 \\
\hline 14 & Olymique de Marsella & 143.50 \\
\hline 15 & AS Roma & 138.50 \\
\hline 16 & Borussia Dortmund & 132.80 \\
\hline 17 & Olympique Lyon & 128.80 \\
\hline 18 & Hamburguer SV & 116.80 \\
\hline 19 & Valencia & 114.00 \\
\hline 20 & Nápoles & \\
\hline
\end{tabular}


Para la experiencia específica del Real Madrid, Blanco y Forcadell (2006), sostienen que la definición de la nueva estrategia de gestión y dirección para el club se sustentó sobre los siguientes pilares

- Reestructuración de la organización interna del club. Consistió en la implantación de una gestión empresarial profesionalizada que se ha estructurado en tres grandes áreas: deportiva, marketing y económico corporativa.

- Potenciación de la imagen de marca del Real Madrid. Se ha dotado a la marca de una proyección mundial que permite su posicionamiento en mercados estratégicos alrededor del mundo.

- Recuperación de activos. Se retomó la gestión de activos que fueron vendidos a otras empresas lo cual ha favorecido a que el valor de esos recursos se haya multiplicado durante los últimos ejercicios a raíz de una gestión y explotación más coherente, planificada y racional.

- Desarrollo de nuevas líneas de negocio que han diversificado las fuentes de ingresos del club.

- Transformar a los seguidores y simpatizantes en clientes efectivos.

Los mencionados autores, afirman que la estrategia del Real Madrid se asienta sobre tres grandes ejes: estrategia deportiva, social y de marketing. Toda la visión estratégica del club está enfocada sobre la base de un doble objetivo: el éxito económico y deportivo ya que no se concibe la posibilidad de lograr el éxito deportivo sin conseguir un buen resultado económico.

Según Ginesta (2011), la expansión del Real Madrid y el Barcelona FC (los dos primeros puestos del ranking citado líneas arriba), se logró a partir de las siguientes acciones:

- Acuerdos de patrocinio con marcas globales.

- Acuerdos paracolaborarcon organizaciones deportivas de otros mercados.

- Acuerdos para emitir contenidos con empresas de comunicación e intensivas en Tecnologías de la Información y la Comunicación (TIC).

- Desarrollode proyectos de Responsabilidad Social Corporativa.

Este autor sostiene que el consumo de productos deportivos está marcado por variables de comportamiento diferentes a las de otros sectores: ante un equipo de fútbol no existen productos sustitutivos y por lo tanto la fidelidad del consumidor (aficionado) hacia su club, no se cuestiona. En este punto se encuentra una de las premisas básicas del nuevo modelo de entretenimiento global promovido por los dos grandes del fútbol español: la voluntad de crear productos de ocio vinculados con una fidelidad incuestionable para sus usuarios, cuyo consumo genera ingresos suficientes para volver a invertir en mejorar el producto principal de la organización, el cual es el equipo. Tanto el Real Madrid como el FC Barcelona conforman un nuevo modelo de multinacionales del ocio, porque al margen del espectáculo futbolístico (exportado y mediatizado a nivel mundial), estas organizaciones estructuran una oferta de productos complementarios a este espectáculo (también productos comunicativos) que son lanzados a un mercado internacional y permiten a los fanáticos, mostrar la pasión por sus colores en todos los ámbitos de su vida cotidiana. En esta línea, Ferrán Soriano (2009) sostiene que el crecimiento de los ingresos de marketing culmina un cambio de modelo fundamental que convierte el negocio de los clubes de fútbol en un negocio de entretenimiento global.

\section{El fútbol peruano}

Se podría afirmar que el fútbol es el deporte más popular en el Perú, aquel que mayor cantidad de gente practica y cuyas incidencias son seguidas con mayor interés. Se percibe que los periódicos deportivos dedican más páginas al fútbol y muchos de sus titulares suelen ser también, de esta disciplina deportiva. Además, es frecuente encontrar restaurantes, cafés, casinos, $\mathrm{u}$ otros establecimientos de gran concurrencia que cuentan con pantallas de televisión que transmiten campeonatos de diversas latitudes. 
Sin embargo, el fútbol peruano actualmente no es el deporte más exitoso del Perú; sitial ocupado por otras disciplinas como el surf, el tiro olímpico o el vóleibol; que son de las pocas disciplinas deportivas en las cuales se ha obtenido algún título de nivel internacional. Los triunfos alguna vez conseguidos en las disciplinas mencionadas, no generan el fanatismo o la reacción popular que las relacionadas con el fútbol.

Seguidamente, para tener un mayor conocimiento del fútbol peruano, se plantea una aproximación al mismo desde los ámbitos deportivo, legal y económico; para luego exponer su funcionamiento organizado.

\section{V.1 Situación deportiva del fútbol peruano}

Para establecer la situación deportiva del fútbol peruano se presentarán en primer lugar, sus logros más importantes tanto a nivel de selección como de clubes; y además, se expondrán sus posiciones a nivel selección y de clubes en los rankings de la FIFA.

Logros más importantes a nivel de selección y de clubes del fútbol peruano han sido las siguientes:

- A nivel de selección: La clasificación a los mundiales de México 70, Argentina 78 y España 82; el título obtenido en la Copa América de 1975, la clasificación a los cuartos de final del Mundial de Corea 2007 de la categoría Sub 17; y el tercer puesto en la Copa América 2011.

La selección peruana ha participado en tres mundiales, siendo México 70 en el que tuvo sus mejores resultados. En Argentina 78, pasó a la segunda ronda en la cual perdió todos sus partidos. En España 82 no pasó de la fase de grupos quedando en el último lugar de su grupo con tan solo dos puntos.
En la Copa América 2011, disputada en Argentina, la selección peruana de fútbol se ubicó en el tercer lugar; su jugador Paolo Guerrero fue el goleador de la misma con 5 goles y Yoshimar Yotún fue nominado a mejor jugador joven del certamen (EI Comercio 2011d).

- A nivel de clubes: Los subcampeonatos en la Copa Libertadores de América (el máximo certamen futbolístico de clubes de la región) del club Universitario de Deportes (1972) y del Sporting Cristal (1997); y el campeonato en la Copa Sudamericana (el segundo torneo en importancia de clubes de la región) de 2003 por parte del club Cienciano del Cuzco'1.

En cuanto a listados o rankings oficiales de fútbol profesional de la Federación Internacional de Fútbol Asociado (FIFA), se pueden observar los siguientes resultados:

- la Selección Peruana de Fútbol se ubicó en la posición 86 en 2009; en la posición 38 en 2010; en la posición 25 en 2011 (luego de su tercer lugar en la Copa América 2011) y en el puesto 47 para agosto de 2012 (FIFA s. f. c).

- Por otro lado, según la International Federation of Football History and Statistics $\left(\right.$ IFFHS) ${ }^{2}$ de la FIFA; el Torneo de Primera División Profesional peruano se ubicó en la posición 24 en el año 2009; en la posición 16 el año 2010; y en la posición 46 el año 2011. Para agosto de 2012, se encuentra en el puesto 39.

- Para agosto de 2012, la misma institución en su ranking top 400 de clubes vigente entre el 1ro de agosto de 2011 y el 30 de julio de 2012, establece que el club peruano mejor ubicado es Universitario

1. La Copa Libertadores de América es el torneo de clubes sudamericanos de fútbol, más importante. Se juega el primer semestre de cada año a partir del año de 1960 y los clubes participantes son aquellos cuyas asociaciones de fútbol que pertenecen a la Conmebol o que forman parte de la Federación Mexicana de Fútbol. La Copa Sudamericana es el segundo torneo de clubes sudamericanos en importancia. Se juega el segundo semestre de cada año a partir del 2002 y los clubes participantes son aquellos cuyas asociaciones de fútbol pertenecen a la Conmebol con la invitación hacia la Federación Mexicana de Fútbol.

2. La IFFHS es una institución que "pretende establecer una documentación cronológica y científica del fútbol mundial y determinar los auténticos records mundiales en todas las áreas del fútbol. Basada en su cualificación universal, la IFFHS también organiza elecciones mundiales y determina rankings mundiales cada año" (IFFHS s. f.). 
de Deportes en el puesto 127 seguido del Juan Aurich de Chiclayo en el puesto 130, Alianza Lima (235), Sporting Cristal (260),CSCD Universidad César Vallejo de Trujillo (287), Sport Huancayo (338), Club Deportivo Universidad San Martín de Porres (350), Club León de Huánuco (351), Club Deportivo Unión Comercio (357),y Club Inti Gas Deportes de Ayacucho (421).

El palmarés mencionado y los resultados en los rankings internacionales de la FIFA, denotan que se han conseguido muy pocos éxitos a nivel internacional lo que lleva a que el fútbol peruano actualmente tenga un reducido prestigio en el extranjero.

\section{V.2 Situación legal}

Para conocer el ámbito legal del balompié peruano en este acápite se presentará lo siguiente: el régimen laboral de los jugadores de fútbol en el Perú, el dilema que configura la transformación a Sociedades Anónimas por parte de los clubes profesionales peruanos, el rol de la SUNAT y los problemas tributarios de los mismos, la deshabilitación de la Federación Peruana de Fútbol de la FIFA acontecida hace algunos años, y el funcionamiento organizativo del fútbol peruano a nivel profesional y amateur.

\section{V.2.1 Régimen laboral de los trabajadores}

En cuanto al régimen laboral de los futbolistas Los contratos laborales de los futbolistas se celebran por escrito entre el club y el futbolista. Estos pueden suscribirse por un periodo de tiempo o por una determinada cantidad de partidos. Ambas partes establecen el monto de la remuneración, los premios por los partidos, seguros, demás conceptos remunerativos y las causas de resolución del acuerdo. Los futbolistas no pueden rescindir su contrato, salvo que el club incumpla con lo acordado por su parte, sino que deben esperar a que éstos concluyan. Tales contratos están regulados por el Estatuto del Futbolista Profesional (EFP) de forma que existe un único formato al respecto y se registran ante la Federación Peruana de Fútbol (FPF) y el Ministerio de Trabajo (MINTRA).
Las obligaciones de los clubes hacia los futbolistas son darle los medios para que reciban atenciones médicas oportunas, proporcionarles raciones alimentarias mientras permanezcan en las concentraciones previas a las competencias y pagarles puntualmente. Si no se cumple con esto último, el club no podrá contar con el futbolista y la institución podrá ser amonestada. Con respecto a las obligaciones de los futbolistas hacia los clubes éstas son: asistir a los partidos, a las prácticas de preparación y a las concentraciones en el lugar y hora señalados; participar en las competencias que el club determine; someterse a la disciplina de sus superiores (comando técnico y autoridades del club) y acatar sus órdenes e instrucciones; y tener en su vida privada un comportamiento compatible con el mantenimiento del eficiente estado físico y mental en su condición de deportista.

Son derechos de los futbolistas el descanso semanal y en días feriados; recibir como mínimo el $20 \%$ del monto total que se pague por su venta a otro club; y no ser excluidos de los entrenamientos ni demás actividades preparatorias del club (salvo que se encuentren lesionados o sancionados). Además de los ya mencionados, pueden acordarse otros beneficios dentro del contrato (El Comercio 2011a).

El problema que se presenta es que muchos de estos derechos no son respetados por los clubes sea por falta de presupuesto o por otro tipo de manejos u omisiones. En esta línea; la Asociación Sindicato de Futbolistas Agremiados del Perú, es una organización que defiende los derechos de los jugadores nacionales de manera que se cumplan sus sueldos y compensaciones acordadas en sus contratos. Esta Asociación es bastante activa y realiza medidas de fuerza cuando sus asociados presentan problemas laborales con los clubes.

\section{V.2.2 El dilema de las sociedades anónimas}


Una Sociedad Anónima «es una sociedad de capitales, con responsabilidad limitada, en la que el capital social se encuentra representado por acciones, y en la que la propiedad de las acciones está separada de la gestión de la sociedad. Nace para una finalidad determinada. Los accionistas no tienen derecho sobre los bienes adquiridos, pero si sobre el capital y utilidades de la misma» (SUNARP s. f.: 1). De esta manera, lo esperado es que todos aquellos que inviertan en acciones de una sociedad anónima tengan objetivos de recuperación de dicha inversión de manera tal que busquen optimizar de la mejor manera el funcionamiento de esta sociedad. Actualmente, la mayoría de clubes de fútbol en el Perú son sociedades civiles sin fines de lucro, y se plantea la posibilidad de establecer una ley que obligue a los clubes de fútbol a convertirse a sociedades anónimas. Este planteamiento ha generado un debate en el medio nacional.

Lo que se pretende, es adoptar una nueva legislación para las sociedades anónimas deportivas, con la cual los clubes de fútbol se verían obligados a convertirse en sociedades anónimas, debe debatirse a partir de tres puntos críticos: i) crisis de liquidez de los clubes, ii) incentivos más favorables para el desarrollo de la actividad $y$, iii) el costo de oportunidad.

En lo que se refiere al primer punto, es una realidad que la mayoría de clubes que participan en el torneo de fútbol profesional no pueden afrontar sus deudas con los ingresos que generan. Se establece así la necesidad de los clubes por inyección de capital y para satisfacerla, éstos deberían adoptar una estructura societaria que permita recuperar el capital. Para conseguir esto, la estructura societaria de Sociedad Anónima es la que se utiliza en el resto de actividades empresariales. Con respecto a los incentivos favorables para el desarrollo de la actividad, una sociedad con fines de lucro tendrá el objetivo de alcanzar el éxito deportivo de manera que se maximizan los ingresos a obtener por cualquiera de los cuatro medios por los que un club puede generarlos (taquilla, publicidad, venta de jugadores y derechos televisivos). En cuanto al tercer y último punto, el costo de oportunidad se refiere a lo que se perdería en caso de que la medida de convertir a los clubes de fútbol en sociedades anónimas fracase. Dada la pobre situación deportiva en la que se encuentra el fútbol peruano, se puede afirmar que el costo de oportunidad no es muy alto (Heeren 2010).

En esta línea, la situación actual del fútbol en el Perú presenta clubes altamente endeudados con el fisco, altos niveles de corrupción en los dirigentes, gestiones económicas deficitarias, pobres resultados deportivos, carencia de nuevos valores, producto fútbol devaluado, nula credibilidad dirigencial e incapacidad para generar nuevos recursos. Todo lo anterior hace que el fútbol en el Perú no sea exitoso comercial y deportivamente hablando. En todos los países en los que el fútbol es un negocio exitoso, la estructura legal de los clubes tiene una lógica corporativa, capitalista o societaria. Convirtiéndose los clubes en sociedades anónimas es que se generarían esquemas de organización de los mismos con propietarios o responsables económicos que tengan interés empresarial, además de ser dirigentes futbolísticos. De esta manera, muchos afirman que la adopción de una nueva legislación para las sociedades anónimas podría tener efectos positivos para el fútbol en el Perú; como los ha tenido en países como España, México, Chile, Colombia, entre otros; que presentaban realidades similares a la peruana (Pizarro 2010).

\section{V.2.3 La SUNAT y los problemas tributarios de los clubes de fútbol profesionales}

La Superintendencia Nacional de Administración Tributaria (SUNAT) «es [...] una Institución Pública descentralizada del Sector Economía y Finanzas, dotada de personería jurídica de Derecho Público, patrimonio propio y autonomía 
económica, administrativa, funcional, técnica y financiera que [...] ha absorbido a la Superintendencia Nacional de Aduanas, asumiendo las funciones, facultades y atribuciones que por ley, correspondían a esta entidad» (SUNAT $s$.f.). Es la entidad encargada en el Perú del cobro de los impuestos a todos los individuos e instituciones que corresponda. Dentro de éstas se encuentran los clubes de fútbol profesionales, así son seis los que presentan deudas con la SUNAT a marzo de 2012.

Seguidamente, se presenta el detalle de la forma de administración de los clubes de fútbol profesional en el Perú, sus deudas a la SUNAT, las deudas a jugadores y si se tratan de sociedades anónimas.

Cuadro 4

Deuda de los clubes de fútbol progresional con la SUNAT y a jugadores a marzo de 2012

\begin{tabular}{|c|c|c|c|c|c|}
\hline $\mathrm{N}^{\circ}$ & CLUB & ADMINISTRA & DEUDA SUNAT (S/) & DEUDA JUGADORES (US\$) & S.A \\
\hline 1 & Universitario & Club & 146 millones & 1.5 millones & NO \\
\hline 2 & Alianza Lima & Club & 26 millones & 570,000 & $\mathrm{NO}$ \\
\hline 3 & Sport Boys & Club & 9 millones & 466,000 & NO \\
\hline 4 & Melgar & Club & 3.5 millones & 56,000 & NO \\
\hline 5 & Cienciano & Club & 2.3 millones & 703,000 & NO \\
\hline 6 & Intigas & Inti Gas S.A.C & 350,000 & 12,000 & SÍ \\
\hline 7 & Cobresol & Club & 0 & 353,000 & NO \\
\hline 8 & José Gálvez & Club & 0 & 84,000 & NO \\
\hline 9 & Cesar Vallejo & Consorcio Vallejo-Sipán & 0 & 58,000 & SÍ \\
\hline 10 & Sport Huancayo & Club & 0 & 1,000 & NO \\
\hline 11 & Real Garcilaso & Club & 0 & 0 & $\mathrm{NO}$ \\
\hline 12 & León de Huánuco & Grupo Jacto-Picón & 0 & 0 & NO \\
\hline 13 & Juan Aurich & Grupo Oviedo & 0 & 0 & SÍ \\
\hline 14 & Unión Comercio & Chávez Import & 0 & 0 & SÍ \\
\hline 15 & Sporting Cristal & Backus \& Johnston & 0 & 0 & SÍ \\
\hline 16 & U. San Martín & U. San Martin de Porres & 0 & 0 & SÍ \\
\hline
\end{tabular}

Fuente: INFOS (2012).

Si los clubes no pagan sus cuentas a la SUNAT, la misma tiene la facultad de embargar sus propiedades (Gestión 2011a). El embargo podría darse para cualquier propiedad, incluso para los logos de los equipos (El Comercio 2011c). De esta manera, si los clubes son sometidos a embargos por la SUNAT, podrían quedarse sin patrimonio.

Cabe mencionar que todos los clubes de fútbol profesionales pueden acogerse a la Ley que promueve la transformación de los mismos en sociedades anónimas, a través de la cual pueden fraccionar sus deudas tributarias y evitar que la SUNAT llegue a tomar medidas extremas como el embargo.
Si bien, esta ley no obliga a los clubes a convertirse en sociedades anónimas, funciona como un incentivo para que lo hagan y puedan mejorar su situación financiera (Gestión 2010).

\section{V.2.4 Deshabilitación de la Federación Peruana de Fútbol (FPF) por parte la FIFA}

El 25 de noviembre de 2008, la FPF fue desafiliada de la FIFA. Esta última alegó «injerencias gubernamentales» por parte del Instituto Peruano del Deporte (IPD) en la FPF, al exigir la renuncia de su presidente Manuel Burga (La República 2008). La FIFA optó por la desafiliación debido a que 
el 13 de marzo del 2006 el IPD acordó la destitución e inhabilitación por cinco años del directorio de la FPF ya que los estatutos de dicha federación no se habían adecuado a los de la Ley del Deporte en el tiempo establecido (Villegas y Sotelo 2007). A pesar de la deshabilitación de Burga por parte del IPD, este fue reelegido en 2006 como presidente de la FPF mientras que la institución gubernamental exigía que éste dejara dicho cargo. La FIFA consideró tal hecho como una «injerencia gubernamental» y por ello decidió desafiliar a la FPF (La República 2008).

La desafiliación de la FPF excluía al Perú de cualquier actividad organizada por la FIFA, sea a nivel de selecciones nacionales o de clubes. Posteriormente, el 20 de diciembre de 2008, la sanción fue levantada condicionalmente por parte de la FIFA, luego de recibir una carta de buenas intenciones firmada por el IPD y la FPF. En la mencionada carta, ambas entidades ponían a conocimiento de la FIFA su intención de trabajar en conjunto para solucionar los problemas que habían tenido y llevaron a la desafiliación. En el comité ejecutivo de la FIFA del 19 y 20 de marzo del 2009, se decidió que se volvería a afiliar definitivamente la FPF a la FIFA si es que esta última pudiera comprobar que las buenas intenciones presentadas en la carta presentada como descargo, se estaban llevando a cabo. Finalmente, este caso llegó a su fin de manera que la FPF volvió a ser afiliada la FIFA.

\section{V.2.5 El funcionamiento organizativo del fútbol peruano}

\section{El Torneo de Primera División Profesional 2012}

El Torneo de Primera División Profesional (TPDP) es un campeonato oficial de la Asociación Deportiva de Fútbol Profesional (ADFP), la cual es una asociación civil sin fines de lucro fundada el 12 de mayo de 1912. Su objetivo principal es la administración a nivel nacional de las actividades deportivas del fútbol profesional en el Perú. Responsable de la organización, supervisión y control de los campeonatos descentralizados (a nivel nacional) de fútbol entre los clubes de la primera división, bajo la supervisión de la Federación Peruana de Fútbol (FPF). Para agosto de 2012, su presidente es el Sr. Luis De Souza Ferreira Salinas (ADFP 2011).

Sin embargo, cabe mencionar que el ente rector del fútbol en el Perú es la Federación Peruana de Fútbol (FPF) la cual fue fundada el 23 de agosto de 1922. La misma se encuentra inscrita en la FIFA y la Conmebol a partir de 1924 y 1925, respectivamente. Se encuentra sujeta al control del IPD y su Presidente es Manuel Burga Seoane (FPF s.f.).

La FIFA (Fédération Internationale de Football Association) es el máximo rector del fútbol en el mundo, su objetivo es «mejorar constantemente el fútbol». Fue fundada en 1904 y su sede se encuentra en Zúrich (Suiza). Para julio de 2011, está compuesta por 208 asociaciones nacionales de fútbol. Su actual presidente es Joseph Blatter de nacionalidad suiza (FIFA s. f. a). Esta asociación cuenta a su vez con confederaciones que la apoyan en dirigir el fútbol en cada continente, las mismas son; Asian Football Confederation (AFC) para Asia, Confédération Africaine de Football (CAF) para África,Confederation of North, Central American and Caribbean Association Football (Concacaf) para América del Norte, América Central y el Caribe; Confederación Sudamericana de Fútbol (Conmebol) para Sudamérica, Oceania Football Confederation (OFC) para Oceanía y Union des Associations Européennes de Football para Europa (UEFA) (FIFA s. f. b).

El Perú al estar ubicado en Sudamérica, es normado por Conmebol, la cual fue fundada el 9 de julio de 1916 y su objetivo es unir, bajo una autoridad común, a las asociaciones nacionales de Sudamérica a fin de lograr el mejor desarrollo y control del fútbol en dicha región (Conmebol s. f. a). Su 
actual presidente es el Dr. Nicolás Leoz, de nacionalidad paraguaya (Conmebols. f. b).

Es importante mencionar que la FPF se encuentra estrechamente relacionada con el Instituto Peruano del Deporte (IPD). El último es la entidad rectora del Sistema Deportivo Nacional (SDN), se trata de un Organismo Público Descentralizado con rango ministerial adscrito al Ministerio de Educación. Su principal función es organizar, planificar, promover, coordinar, evaluar e investigar a nivel nacional el desarrollo del deporte, la recreación y la educación física en todas sus disciplinas, modalidades, niveles y categorías (IPD s. f.).

La FPF es una de las federaciones que el IPD supervisa. Es por esto que la FPF depende del IPD para la aprobación de sus proyectos, para dicho fin la FPF debe cumplir ciertas obligaciones como cumplir los requisitos de la ley peruana. A su vez, como la FPF es una de las federaciones afiliadas a la FIFA debe también cumplir con la normativa de esta última. La FPF se encarga de la gestión de todo lo concerniente a las selecciones peruanas de fútbol y de los torneos internacionales en los que participen los equipos peruanos de fútbol. Finalmente, la ADFP se encarga, por pedido y supervisión de la FPF, de la organización de todos los campeonatos de fútbol internos del país.

\section{Equipos participantes y bases generales}

Según la ADFP (2012), los equipos participantes del Torneo de Primera División Profesional 2012 son los siguientes dieciséis: Alianza Lima, Cienciano, Cultural, Social y Deportivo, Universidad César Vallejo SAC, Deportivo Cobre Sol FBC, Deportivo Universidad San Martín de Porres S.A., Deportivo Unión Comercio S.A., F.B.C. Melgar, Inti Gas Deportes, José Gálvez FBC, Juan Aurich, León de Huánuco, Real Atlético Garcilaso, Sport Boys Association, Sporting Cristal, Sport Huancayo, y Universitario de Deportes.

El torneo consta de tres etapas. La primera de dos «ruedas» con partidos de «ida y vuelta»; la segunda constará de las «liguillas» A y B, esta etapa constará de dos grupos de 8 equipos cada uno, los cuales se enfrentaran en dos «ruedas» con partidos de «ida y vuelta». La tercera etapa se denomina «play off», en ésta se disputarán dos partidos para definir al Campeón Nacional y al Sub Campeón Nacional entre los equipos que hayan resultado en el 1er lugar de ambas las Liguilla. Específicamente, se tiene:

Cuadro 5

ADFP: Etapas del torneo descentralizado 2012

\begin{tabular}{|l|l|}
\hline Etapa & Especificaciones \\
\hline Primera & $\begin{array}{l}\text { La primera etapa se jugaran en } 30 \text { fechas, cada uno con partidos todos contra todos en dos ruedas. } \\
\text { El Campeón de la Primera Etapa clasificara a la Copa Libertadores } 2013 \text { como Perú 3, siempre y cuando quede } \\
\text { dentro de los ocho primeros lugares de la Tabla de Posiciones al finalizar el torneo. }\end{array}$ \\
\hline Segunda & $\begin{array}{l}\text { Se jugará al finalizar la segunda «rueda» de la Primera Etapa. Aquí, ocho clubes conformarán la «liguilla» A y el } \\
\text { mismo número la «liguilla»B. }\end{array}$ \\
$\begin{array}{l}\text { La conformación de las «liguillas» se realizará mediante sorteos que serán formados por parejas, sin considerar } \\
\text { ningún parámetro adicional, de la siguiente manera: } 1 \text { y } 2 ; 3 \text { y } 4 ; 5 \text { y } 6 ; 7 \text { y } 8 ; 9 \text { y } 10 ; 11 \text { y } 12 ; 13 \text { y } 14 ; 15 \text { y } 16 .\end{array}$ \\
$\begin{array}{l}\text { Ambas Liguillas se jugaran de forma paralela en dos ruedas y cada rueda constará de siete Fechas y los equipos } \\
\text { ingresarán con el puntaje que obtuvieron en la Primera Etapa. Resultará ganador de cada grupo, quien obtuviera } \\
\text { el mayor puntaje en cada uno de ellos. Si hubiera un empate de dos equipos para definir el primer lugar de } \\
\text { las Liguillas A ó B, se jugará un partido definitorio dentro de las } 72 \text { horas. Si el partido terminara empatado se } \\
\text { jugará inmediatamente dos tiempos suplementarios de } 15 \text { minutos cada uno. Si al término de los dos tiempos } \\
\text { suplementarios continuara el empate, se lanzarán penales de acuerdo al sistema FIFA hasta definir un ganador. Si } \\
\text { empataran tres o más equipos en el primer lugar se aplicará el Artículo } 13^{\circ} \text { y los dos mejores ubicados definirán al } \\
\text { ganador. El partido mencionado de desempate se juega en un campo neutral. }\end{array}$ \\
\hline
\end{tabular}




\begin{tabular}{|l|l|}
\hline Etapa & Especificaciones \\
\hline Tercera & $\begin{array}{l}\text { Se desarrolla con los equipos que ocupen el primer lugar de la «liguilla» A y de la «liguilla B», ambos jugarán dos } \\
\text { partidos («ida y vuelta») y el ganador será el Campeón Nacional, el Club perdedor será proclamado Sub-Campeón }\end{array}$ \\
& $\begin{array}{l}\text { Nacional. } \\
\text { Si después de los } 2 \text { partidos no se definiera al Campeón Nacional se jugará un partido definitorio dentro de las } 72 \\
\text { horas en un campo neutral. }\end{array}$ \\
\hline
\end{tabular}

Elaboración propia sobre la base de Torneo Descentralizado 2012 Copa Movistar (ADFP 2012).

Los Clubes que ocupen del tercero al décimo sexto lugar en el puntaje acumulado de las dos Etapas, serán proclamados como tales en la tabla de posiciones final. Los Clubes que ocupen el $15^{\circ}$ y $16^{\circ}$ lugar en el puntaje acumulado después de la segunda Etapa, descenderán de categoría.

Los Clubes clasificados a Copa Libertadores 2013 son: el Campeón Nacional, el SubCampeón Nacional, el equipo que haya ocupado el tercer lugar, el club que ocupe el primer lugar de la primera etapa del torneo. Los tres clasificados a la Copa Sudamericana 2013 son aquellos que ocupen los puestos inmediatos, después de los tres Clubes clasificados a la Copa Libertadores. El Campeón de la Copa Inca será el cuarto clasificado a la Copa Sudamericana.

\section{El fútbol amateur en el Perú}

En el Perú, los principales torneos amateur que existen son el Torneo de Segunda División Nacional (TSDN) y la Copa Perú. Como son campeonatos amateur, no es obligatorio sus equipos para poder jugar Los equipos del torneo de fútbol profesional que pierdan la categoría, descenderán al TSDN.

El TSDN es un campeonato de 12 equipos en el que todos juegan entre sí haciendo un total de 22 fechas, cada equipo jugará 11 fechas de local y 11 fechas de visitante. Hasta el año 2005 participaban solo equipos de Lima y Callao pero a partir del 2006 se empezó a permitir la participación de equipos de todos los departamentos del país. El campeón de este torneo, asciende directamente a la primera división del fútbol profesional peruano. Por su parte, los dos equipos que queden últimos en el

TSDN jugarán la vigente edición de la Copa Perú desde la etapa Regional.

La Copa Perú es un campeonato de clubes divididos en ocho regiones según el departamento del Perú al que pertenecen. Consta de cinco etapas: Distrital, Provincial, Departamental, Regional y Nacional. En las cuatro primeras etapas todos los equipos juegan dos veces entre sí: cada uno una vez de local y la otra de visitante. El equipo que campeona la etapa Distrital clasifica a la etapa Provincial, el que campeona la Provincial clasifica a la Departamental, el que campeona la Departamental clasifica a la Regional y el campeón y subcampeón de la Regional clasifican a la Nacional. Dado que son ocho regiones y clasifican dos por región se tiene un total de 16 equipos participando desde los Octavos de Final de la etapa Nacional. En dicha etapa el sistema es el de eliminación simple (el equipo que pierde en los dos partidos en su conjunto, es eliminado) con partidos de local y visitante para cada equipo, es decir, un total de dos partidos por fase. De esta manera, se llega a la final de la Copa Perú, y el campeón de ella asciende al Torneo Profesional mientras que el subcampeón jugará la siguiente edición del TSDN. Los equipos que quedan eliminados de este torneo pueden volver a participar de ella en su siguiente edición.

\section{V.3 Situación económica}

No se puede dar una cifra exacta sobre cuánto dinero mueve el fútbol peruano, sin embargo, existen montos aproximados. Carlos Capio, (ex gerente de fútbol de Alianza Lima) dio la cifra de US\$22 millones para el 2009; Germán Leguía, (ex gerente deportivo de Universitario de Deportes), 
lanzó una cifra de US\$30 millones para el 2009 y que este monto aumentaría en $15 \%$ para el 2010, es decir, US\$39 millones para dicho año (Enfoque económico 2010); Julio Pacheco, ex presidente de Universitario de Deportes, dio una cifra de US\$25 millones para el 2009 y US\$30 millones para el 2010 (Rosado 2011). Pacheco también declaró que el negocio del fútbol en el Perú se encuentra financiado en aproximadamente un $90 \%$ por auspicios de empresas como Coca-Cola, Cristal, Direct TV, Hyundai, LAN, Nike, Telefónica, Umbro, entre otras. Además de que el financiamiento podría ser mucho mayor si es que más empresas invirtieran en este negocio; sin embargo, se cree que no se atreven a hacerlo debido a la manera tan irregular en la que se manejan los clubes en el país (Rosado 2011). Así mismo, Raúl Castro (Gestión 2011b) afirma que el fútbol peruano mueve aproximadamente 30 millones de dólares en su artículo Dos jugadores en el Perú cobran US\$ 50 mil mensuales (Entrevista a Francesco Manassero, presidente de la Agremiación de Futbolistas) afirma que: «¿Cuánto dinero mueve el fútbol peruano?, algunos hablan de US\$ 30 millones, basándose en el dinero que paga la TV a los clubes, así como los auspicios».

Las fuentes de ingresos de los clubes profesionales son cuatro: los derechos de transmisión televisiva, la publicidad, la venta de jugadores, y las taquillas recaudadas. Según el mencionado Julio Pacheco, éstas representan el 60\%, 25\%, 5\% y $10 \%$ respectivamente, de los ingresos totales de los mencionados clubes (Rosado 2011).

No se tiene información exacta acerca de los ingresos por derecho de transmisiones televisivas. Así, se pueden citar algunas cifras referenciales. En este sentido, se dice que para el 2011, el club Universitario de Deportes no firmó contrato alguno (Netjoven 2010b). Por su parte, el Cienciano del Cuzco generó ingresos anuales por US\$ 650 mil desde el 2006 hasta el 2009; firmando un contrato para el 2010 y 2011 por US\$ 900 mil anuales y desde el 2012 hasta el 2015 estos se dice que serán de US\$1 millón anuales (Líbero 2011c).

Los clubes generan ingresos por publicidad principalmente por la colocación de logos de distintas marcas en sus camisetas, tampoco se manejan montos exactos al respecto pero, en la prensa se encontraron cifras que especulan con aproximados. Así, se tiene que el año 2009, Universitario de Deportes recibió US\$ 1 millón por colocar el logo del Grupo Santo Domingo en la parte delantera de su camiseta. En 2010, se dice que Alianza Lima recibió US\$ 1 millón por colocar el logo de Cerveza Cristal en el pecho de su camiseta, US\$ 600 mil por el logo de Banco Azteca en la parte posterior de la misma, y US\$ 70,000 por el logo de la marca de ropa Pieers en sus mangas. Asimismo, la Universidad San Martín recibió US\$200 mil por poner el logo de Herbalife en frente de su camiseta, US\$1 millón por el logo de la compañía de cable Direct TV en una manga de la misma (por tres años) y el logo de Cosapi Data en la otra manga por brindarle computadoras por un valor de US\$ 80 mil a US\$ 100 mil. El Grupo Oviedo le da US\$ 500 mil a Juan Aurich por poner su logo en el dorso (El Comercio 2010b).

La mayoría de transferencias en el TPDP se hace sin costo alguno ya que, por lo general, éstas se hacen cuando los jugadores han terminado el contrato que los vincula con su club anterior. En 2009, hubo 403 transferencias; en 2010 después de terminada la fase general hasta el final de dicho año hubo 107 transferencias y hasta antes de las «liguillas» de 2011 hubo 360 transferencias (De Chalaca 2011). Las transferencias que involucraron una operación financiera entre clubes fueron, por lo general, aquellas en las que el jugador involucrado tenía como destino o procedía de un club extranjero. No se tiene cifras confiables acerca de los montos que se mueven en cuanto a las transferencias de los jugadores.

Un dato adicional e interesante es que según Francesco Manassero (Presidente de la Asociación de Futbolistas Agremiados del Perú), en cuanto al nivel de sueldos, el fútbol profesional peruano está en la región luego de Brasil, Chile y Ecuador; así los mismos van de S/ 1,000.00 (mil nuevos soles) a US\$ 50,000 (cincuenta mil dólares americanos); habiendo dos jugadores con este último sueldo mensual (Gestión 2011b).

\section{Taquillas recaudadas en los partidos}

En el siguiente cuadro, se puede observar los ingresos por taquillas totales entre el año 2009 y el 2011 del Torneo de Fútbol Profesional en el Perú. 


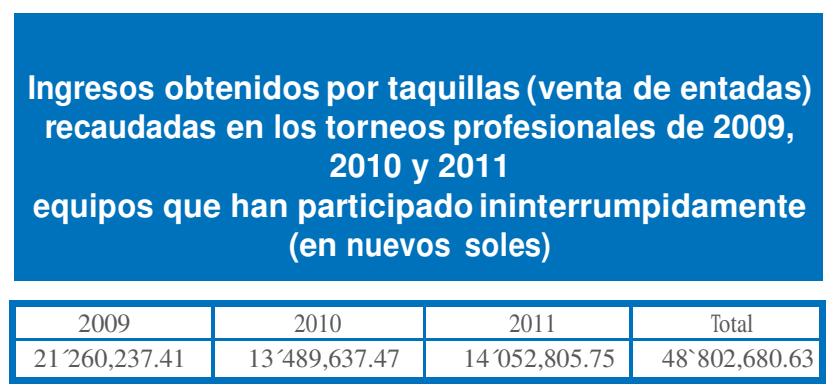

Fuente: ADFP y FPF.

Elaboración propia.
En el siguiente cuadro, se presenta un ranking de ingresos por taquillas, puntos obtenidos y ubicación en la tabla de posiciones de los equipos que participaron en el TPDP 2009, 2010 y (sólo se han considerado a los equipos que han participado de forma ininterrumpida en todo el período de análisis):

\begin{tabular}{|c|c|c|c|c|c|c|c|c|c|c|}
\hline E quipa & $\begin{array}{c}2009 \\
\text { In gliewos is? }+ \text {; }\end{array}$ & Puntos & 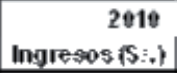 & Puntos & $\begin{array}{r}2011 \\
\text { Ingiesos }(\mathbf{S}:+\mathbf{t}\end{array}$ & Puntos & $\begin{array}{c}\text { Total de Ingre sos } \\
\text { iS: }\end{array}$ & $\begin{array}{l}\text { Toual de } \\
\text { Puntos }\end{array}$ & $\mathbf{T I}$ & Tr \\
\hline Alianza Atlético & $390,549.45$ & 46 & $392,301.16$ & 44 & $300,762.46$ & 33 & $1,083,613.07$ & 123 & 12 & 11 \\
\hline Alianza Liına & $0,796,754.01$ & 75 & $2,305,04030$ & 70 & $0,007,217.95$ & 57 & $10,069,011.74$ & 210 & 1 & 1 \\
\hline Ciençisno & 1.125 .90193 & 57 & 933.09234 & 47 & $1.802,336 \mathrm{EE}$ & 40 & 3.866 .33343 & 144 & 5 & 9 \\
\hline $\begin{array}{c}\text { Colegio Nacional de Iquitos } \\
\text { Inti Gas }\end{array}$ & $\begin{array}{l}2,373,306.44 \\
1,949,99403\end{array}$ & $\begin{array}{l}50 \\
51\end{array}$ & $\begin{array}{c}1,289,33205 \\
931,527+2\end{array}$ & $\begin{array}{l}56 \\
56\end{array}$ & $\begin{array}{l}445,263.91 \\
\text { S.E. } 2, E .471\end{array}$ & $\begin{array}{l}33 \\
44\end{array}$ & $\begin{array}{l}4,108,422.41 \\
3,444,176,16\end{array}$ & $\begin{array}{l}139 \\
161\end{array}$ & $\begin{array}{l}4 \\
7\end{array}$ & $\begin{array}{c}10 \\
7\end{array}$ \\
\hline Juan Aurich & $2,165,53901$ & 71 & 931,76652 & $\mathrm{E} 8$ & $1,782,71503$ & 57 & $4,880,020.56$ & 196 & 3 & 3 \\
\hline Ma olgor & $1, n 00,00289$ & 55 & $57 A, 11 A 7 R$ & an & RQว,กAORก & 34 & $2,156,1582 A$ & 120 & 10 & 10 \\
\hline Sport Huancayo & $1,827,421.49$ & 67 & 373,24030 & 59 & $760,719.20$ & 50 & $3,481,381.49$ & 176 & 6 & 5 \\
\hline Sporting Cristal & $355,4.35 .11$ & 57 & $1,352,53714$ & 6.9 & 931,860132 & 38 & $3,240,993.57$ & 159 & 8 & 8 \\
\hline Universidad C èsar Vallejo & $1,385,168.00$ & 66 & 880,84316 & 70 & $905,230.09$ & 34 & $3,171,241.25$ & 170 & 9 & 6 \\
\hline Universidad San Martın & 182.33135 & 57 & $1,111,13204$ & $G$ & 148.53852 & 97 & $1,99:, 1011.9 z$ & 208 & 11 & 2 \\
\hline I Iniversitarin de Depnntes & $4, \cap \cap 5,7817 \cap$ & 81 & $1,779,1 \cap 91.9$ & 77 & $1,823,4.3590$ & .34 & $7,559,32679$ & 187 & 2 & 4 \\
\hline Total & $21,260,237.41$ & 755 & $13,489,637.47$ & 756 & $14,052,805.75$ & 501 & $48,802,680.63$ & 2012 & & \\
\hline Promedio & \multicolumn{2}{|c|}{$1,771,686.45$} & \multicolumn{2}{|c|}{$1,124,136.46$} & \multicolumn{2}{|c|}{$1,171,067.15$} & $4,066,890.05$ & 167.6667 & & \\
\hline
\end{tabular}

Fuentes: ADFP, FPF, Data Sport, www.dechalaca.com. TI: Puesto en función al Total de Ingresos / TP: Puesto en función al Total de puntos acumulados.

Revisar las taquillas y los resultados deportivos de los clubes profesionales de fútbol en el Perú en el período 2009-2011, permite observarlo reducidos que son los ingresos de los equipos por la venta de entradas. En segundo lugar, se puede afirmar que muchas veces los resultados deportivos van de la mano con la recaudación por taquillas aunque puede haber equipos con campañas deportivas destacadas o exitosos y con recaudaciones poco auspiciosas (el Club Deportivo Universidad San Martín de Porres es un ejemplo d esto). En tercer lugar, los equipos con mayor tradición e hinchada son los que mayores recaudaciones presentan sobre la base de un gran número de seguidores a nivel nacional. Finalmente, llegar a los «play off» puede ser una «tabla de salvación» económica para los equipos que llegan a disputarlos.

\section{Los problemas del fútbol peruano}

Existe un diagnóstico muy serio desarrollado por la firma peruana Apoyo Consultoría en 2004, titulado Desarrollo e implementación de una estrategia para la mejora de la competitividad e incremento de la rentabilidad en el fútbol peruano, el mismo fue encargado por la FPF. Éste plantea que «en general, la informalidad observada en las relaciones entre los entes y la evolución individual y poco coordinada de cada uno de ellos ha generado una situación en la que existen pocas iniciativas grupales y se hace difícil el desarrollo de una estrategia conjunta. Se podría decir que los principales problemas que enfrentan los entes del fútbol peruano son: i) No existen asignaciones formales de responsabilidades básicas para el funcionamiento del sistema, ii) No 
existen relaciones formales entre los entes, y iii) No existen funciones asignadas relacionadas con el desarrollo del fútbol profesional de alto nivel. La credibilidad y la confianza del público en los entes del fútbol es pobre» (Apoyo Consultoría 2004: 84). Según este estudio para junio de 2004, el fútbol peruano patrimonialmente se encontraba técnicamente en quiebra y los ingresos que el fútbol generaba por concepto de taquillas, al igual que la asistencia a los partidos, presentan una tendencia decreciente sobre la base de lo sucedidoentre el año 1998 y el 2003.

Por otro lado, el fútbol peruano presenta inconvenientes como: i) altos niveles de corrupción en los dirigentes, ii) gestiones económicas deficitarias, iii) pobres resultados deportivos, iv) carencia de nuevos valores, v) el producto fútbol está devaluado, vi) nula credibilidad dirigencial, e vii) incapacidad para generar nuevos recursos (Pizarro 2010).

Para 2004, se afirma que si se consolidasen los estados financieros de todos los clubes participantes en la primera división de fútbol del Perú, se obtendría un pasivo de US\$ 26 millones y un patrimonio de US\$2 millones, es decir, se observaría un altísimo nivel de apalancamiento (Heeren s. f.).Así, se afirmaba que para dicho año, el fútbol peruano tenía deudas que duplicaban los ingresos brutos anuales de la industria y se estimaba que éste pierde más de US\$ 2 millones al año (Apoyo Consultoría 2004). En esta línea, las taquillas presentaron una tendencia decreciente de 1998 al 2003, solo con un incremento para el año 2002, lo que impactó directamente en los ingresos generados (Heeren s. f.).

Otras conclusiones muy importantes del mencionado informe de Apoyo Consultoría (2004) que grafican la problemática del fútbol peruano, son las siguientes:

- Los pobres resultados deportivos y el producto fútbol devaluado son los principales causantes de que la asistencia a los estadios fuera mínima, había una percepción de bajo nivel del torneo local. Existía una disparidad entre los equipos tanto del torneo profesional como en los torneos amateur, sobre todo de estos últimos, que hacía que la incertidumbre por los resultados disminuyera. Otro de los motivos que generaba esta taquilla decreciente era la violencia, mucha gente dejaba de ir a los partidos ya que consideraban los estadios como lugares peligrosos. Esta cada vez menor asistencia de gente a los estadios, redujo el interés de los patrocinadores, por lo que disminuyen los ingresos lo que genera la incapacidad para generar nuevos recursos.

- Las gestiones económicas deficitarias y la incapacidad para generar nuevos recursos se debían principalmente a la falta de orientación de los clubes hacia la generación de ingresos de forma transparente a partir del producto fútbol. Para el 2004 el público creía que los clubes que fueran administrados por gerentes profesionales y como empresas lograrían buenas campañas deportivas. Otro factor es que el sistema del torneo amateur de la Copa Perú no permite que los clubes puedan generar una institucionalidad que les dé una base económica si es que llegan a primera división. A partir de las gestiones económicas deficitarias y la incapacidad para generar nuevos recursos es que los clubes se endeudan con el fisco.

- La carencia de nuevos valores surgía de los problemas en las divisiones menores, los dos principales eran el reducido nivel de desarrollo de canteras en los clubes, y la falta de una estructura continua que soporte todas las etapas de formación de un futbolista. A 2004, eran dos los clubes que monopolizaban todos los títulos en los campeonatos de divisiones menores, se notaba así una disparidad entre el nivel de los equipos participantes en los torneos de menores. Esto hacía que dichos equipos jueguen a lo largo del año muy pocos partidos que les exijan un esfuerzo superlativo, lo que se reflejaba en el bajo nivel de competencia de las selecciones peruanas juveniles ya que la mayoría de convocados provenían de tales clubes (Apoyo Consultoría 2004). 
- Los altos niveles de corrupción en dirigentes y la nula credibilidad dirigencial se encontraban relacionadas directamente con el sistema de justicia y los entes del fútbol que existen en el Perú. En lo que se refiere al sistema de justicia, existen tres entidades que la administran: Comisión de Justicia de la Asociación Deportiva de Fútbol Profesional, Comisión de Justicia de la Federación Peruana de Fútbol y los Consejos Distritales; cada una de estas tiene sus propias reglas. De esta manera, las reglas de una pueden anular a la otra y se generan malos entendidos en los procesos de justicia que permiten que los problemas se resuelvan por influencias personales de los dirigentes de los equipos (estén o no involucrados en el problema). En cuanto a los entes del fútbol peruano, son tres los involucrados en el desarrollo del fútbol en el Perú: el Instituto Peruano de Deporte (IPD), la Federación Peruana de Fútbol (FPF) y Asociación Deportiva de Fútbol Profesional (ADFP). El problema surgía con las dos últimas ya que éstas no coordinaban objetivos de trabajo y no se encontraban vinculados a través de contratos formales, es decir, abundaba la informalidad.

Para tener un mayor acercamiento a la problemática del fútbol peruano, se desarrollaron una serie de entrevistas desarrolladas a periodistas y dirigentes del fútbol peruano. De la aplicación de las mismas, se puede afirmar que los problemas del mencionado fútbol son los siguientes:

- La carencia de planificación a todo nivel y la presencia de una visión de corto plazo en la mayoría de los dirigentes de los clubes de fútbol. Así, por ejemplo se prioriza ganar el campeonato profesional y se deja de lado proyectos institucionales con objetivos de largo plazo.

- No existe una institucionalización de los clubes, estos no son seriamente gestionados. La mayoría de los clubes no funcionan como organizaciones estructural y administrativamente.
- Falta de una adecuada profesionalización de los dirigentes y por ende, de los clubes. Esto se refleja en una gran improvisación en el fútbol peruano.

- Desorganización del fútbol peruano a todo nivel. Los clubes y las instituciones no presentan una adecuada organización.

- Ausencia de proyectos de trabajo con las divisiones menores de los clubes. Son muy pocas las instituciones que apuestan seriamente al trabajo con menores.

- Ingentes deudas de los clubes de fútbol profesional. Debido a las malas gestiones, los clubes sostienen actualmente grandes deudas con la SUNAT, éstas son de sumas tan fuertes que algunos afirman que sería imposible su cancelación.

- Informalidad de muchos jugadores de fútbol profesional que no se cuidan adecuadamente según su condición de deportistas del alto rendimiento.

- Falta de preparación psicológica de los futbolistas. Esta parte es dejada de lado en la gran mayoría de las instituciones deportivas.

- Excesivo rol del periodismo en la dinámica del fútbol peruano (éste tiende a crear demasiada expectativa en la calidad, rendimiento y potencial de los jugadores y además, los medios de comunicación se enfocan en informar acerca de temas extra deportivos).

- Falta de compromiso de algunos jugadores con la selección nacional. Algunos afirman que jugadores de la selección no se comprometen adecuadamente con el equipo nacional lo que influye en la falta de clasificaciones a los mundiales de fútbol.

- Falta de profesionales especializados en el fútbol a todo nivel. Desde lo administrativo, la gestión, lo médico, entre otros; no existen muchos profesionales especializados que trabajen en los clubes de fútbol.

- La presencia de corrupción en diferentes estamentos del fútbol peruano. 
- Falta de asistencia a los estadios lo que hace que una de sus fuentes de ingreso sea muy reducida.

- Los empresarios deben invertir en equipos tradicionales para solidificar la identidad del fútbol peruano. Algunos afirman que al dispersarse la inversión en muchos clubes que no tienen mayor cantidad de fanáticos, la identidad del fútbol peruano no se consolida.

Adicionalmente, los entrevistados mencionaron que un club peruano que puede servir de ejemplo -en cuanto a su gestión deportiva y administrativa-, era el Club Deportivo Universidad San Martín de Porres.

Como se puede apreciar, el fútbol peruano presenta una serie de graves problemas. Éstos se presentan a nivel de las instituciones que lo dirigen, de los clubes profesionales que participan en el torneo de primera división, de los medios de comunicación, de los propios jugadores, entre otros. Sin embargo, a pesar de esta situación complicada, existen algunos esfuerzos que apuestan al desarrollo del fútbol peruano como opción de entretenimiento, deporte saludable para la población, o como negocio. Un esfuerzo interesante a nivel de los clubes fue el nombrado por los entrevistados, experiencia que se presenta a continuación.

\section{Conclusiones y recomendaciones}

Desde hace varias décadas, el fútbol peruano se encuentra en estado de emergencia. Su problemática se presenta tanto a nivel de los entes que lo manejan, de los clubes profesionales, de los propios jugadores, de su ámbito profesional y también a nivel amateur. Sin embargo, El fútbol tiene un gran potencial en el Perú debido a la gran acogida popular de este deporte. Este país se encuentra ávido de victorias y festividades que potencien y consoliden su identidad. Así, ante la falta de victorias deportivas, una selección de fútbol con éxitos o clubes ganadores; es que la gastronomía ha tomado el lugar de elemento catalizador de la peruanidad. Así, por ejemplo, en un artículo de la revista GQ Latinoamérica publicado en julio de 2011, se afirma que «los peruanos no tienen futbolistas galácticos ni estrellas que brillen en Hollywood, pero tienen cocineros. Son los personajes más queridos del país, la prensa sigue sus pasos y meten golazos de media cancha cada vez que renuevan una carta o abren un nuevo restaurante [...] En una país donde la frase "barriga llena, corazón contento" podría estar tranquilamente en la Constitución o el himno nacional y donde los chicos quieren ser cocineros y no futbolistas - según dijera en una entrevista Ferrán Adriá [...] (GQ Latinoamérica 2010)». Así, en el caso peruano se puede afirmar que el sitial que tiene el fútbol en países como Brasil, Argentina o Uruguay, ha sido ocupado por la gastronomía.

Sin embargo, la obtención del tercer lugar en la Copa América 2011 desarrollada en la Argentina con celebraciones desaforadas que ningún otro deporte o actividad en el Perú produce, permite afirmar que el fútbol tiene un sitial de amalgama o conglomerado de la identidad nacional. La presencia de jugadores con una actitud y características inusitadas (aguerridos, luchadores, con carácter y ganadores) en los últimos años en el Perú como Paolo Guerrero, ha hecho que muchos peruanos se vuelvan a identificar con la selección nacional de fútbol que a pesar de representar a una federación, la mayoría asume que los representa como nación. Así, una recuperación del fútbol peruano no debe basarse sólo en el trabajo en aspectos técnicos y físicos sino también, en una labor psicológica que potencie el rendimiento de los jugadores, la formación de su carácter y por ende, la identificación de la ciudadanía con la selección nacional y el fútbol en general.

Queda claro, que sin una labor con las divisiones menores, cualquier esfuerzo será insuficiente ya que las actuales generaciones de jugadores que militan en la primera división o están cercanos a ella, tienen talento 0 habilidades naturales pero, la gran mayoría de ellos no ha recibido una formación adecuada en ningún sentido. El trabajo con menores implica una seria planificación que respete la visión de largo plazo y que privilegie el desarrollo del fútbol peruano más allá de los intereses de cualquier tipo. 
Por otro lado, muchos expertos afirman que la conversión a Sociedades Anónimas es la gran solución para el logro de la gestión adecuada de los clubes profesionales de fútbol. Sin embargo, en la práctica se puede observar que dos de las instituciones más importantes del mundo como el Real Madrid y el Barcelona FC, no son sociedades anónimas y son asociaciones sin fines de lucro. Más que el régimen societario, lo más importante es que profesionales responsables, capacitados y con un código de ética adecuado asuman las riendas de los entes que manejan el fútbol y de los clubes que participan en el torneo profesional. Esto producirá que los clubes trabajen con planes a largo plazo como los del primer mundo y con objetivos económicos, deportivos y sociales bastante claros. En esta línea, la Universidad San Martín de Porres constituye un ejemplo a seguir por todos los equipos de fútbol profesional nacionales. Sólo con ápices estratégicos profesionales y éticamente adecuados es que el fútbol peruano podrá salir para adelante. Así, por ejemplo, queda la desconfianza frente a refinanciamientos de deudas tributarias cuando las dirigencias de los clubes no cuentan con el respaldo que genera la confianza.

Otro tema muy importante es que una opción que podría evaluarse es que el torneo y la selección sean manejados por una misma institución. En ese caso, la ADFP y la FPF, tienen propios intereses $y$ organizaciones de forma que se redunda $y$ se obstruyen sus labores mutuamente. Una selección y un torneo manejados por una sola organización podrían facilitar el crecimiento del fútbol peruano.

Se debe considerar que el fútbol amateur es extremadamente informal y desorganizado, así es conocida la presencia de trifulcas descomunales en los partidos de la Copa Perú y de los torneos amateur en general. Es desastroso para el desarrollo del fútbol peruano que sus bases sean tan endebles y poco serias.

Lo anterior también está relacionado con la inseguridad ciudadana en la que se desarrolla el Perú, la cual está presente en todo ámbito de la sociedad, incluido el deporte y por ende, en los estadios de fútbol. La inseguridad que ronda en los mismos hace que muchos peruanos evalúen seriamente su asistencia a los partidos de fútbol y que opten por alternativas de entretenimiento mucho más seguras.

Para ser sostenibles, las empresas necesitan fuentes de ingresos. Las de los clubes de fútbol profesional se encuentran distribuidas entre los derechos de transmisión por televisión, la publicidad diversa, la venta de jugadores, y las taquillas recaudadas en los partidos. Todos estos ingresos se ven subvalorados por el poco interés de la población en el fútbol como espectáculo. A esto se puede adicionar la gran presencia de productos sustitutos al fútbol en la industria del entretenimiento en general de forma que los consumidores se deciden por alternativas más atractivas, seguras y que les generan un mayor valor agregado en todo sentido.

En el ámbito de los ingresos por taquillas, debe tomarse en cuenta que el factor que más las potencia es el desempeño deportivo de los equipos. En esta línea, los dos equipos que más recaudaron por taquillas en los campeonatos profesionales entre 2009 y 2011; fueron Alianza Lima y Universitario de Deportes. Además, el primero fue el club que mejor campaña deportiva desarrolló a nivel del torneo nacional sin embargo, no llegó a ser campeón nacional en ninguno de los tres campeonatos. El CNI de Iquitos es el cuarto equipo con mayor cantidad de ingresos en el período analizado, cabe destacar que se trata de un equipo con historia deportiva y tradición de manera que sí se combinan los años de trayectoria y la gran cantidad de fanáticos, se puede encontrar grandes posibilidades para el fútbol en provincias. Por otro lado, el quinto puesto es ocupado por Cienciano del Cusco, el sexto está en manos de Sport Huancayo y el séptimo es el Inti Gas de Ayacucho. Todos son equipos de provincias, lo que puede aunarse al potencial económico de las mismas que se encuentran creciendo de forma sostenida lo que, por ejemplo, se puede observar en el crecimiento del retail moderno y en ingreso per cápita de ellas.

Cabe mencionar que el jugar los «play off» impacta muy positivamente a los ingresos de los 
equipos. Así, lo recaudado en dichos partidos por los equipos participantes en estos encuentros deportivos, representa porcentajes de entre $20 \%$ y $70 \%$ de sus ingresos anuales por taquillas por lo que el llegar a este partido final puede ayudar económicamente a los equipos. Así, el poder jugar este encuentro puede tornarse en el principal objetivo de muchos equipos participantes.

Se quiere cerrar este estudio afirmando que una buena gestión administrativa no tiene mayores secretos. Se parte de un plan estratégico que debe ser implementado y controlado de forma eficiente además, de los resultados económicos que el mencionado plan debe generar para la sostenibilidad de la organización sobre la base de una visión, misión, objetivos, estrategias y políticas claras. No es necesario «inventar la pólvora» en la gestión organizacional ni deportiva; en esta línea, se puede revisar el marco teórico de este trabajo con ideas concretas que han funcionado exitosamente haciendo que el deporte y el fútbol sean modelos de negocio rentables. Todo debe ser diseñado y gerenciado por personas con las competencias profesionales y humanas idóneas. Solo así, el fútbol peruano será algún día un proyecto sostenible, sano y rentable. 


\section{BIBLIOGRAFÍA}

\section{AFP INTERNACIONAL}

2011 «Uruguay exportó 1.414 futbolistas en 10 años». En: redacción.180.com.uy. 04/01/2011. Fecha de consulta: 30/07/2011. <http://redaccion.180. com.uy/articulo/16151_Uruguay-exporto-1414futbolistas-en-10-anos>

2004 «Beckham and Real Madrid Asia-bound again». En: Agencias Pekín, 16 de enero del 2004.

ALTUVE, Eloy

2011 «Consolidación del modelo deportivo globalizador transnacional». En: Espacio Abierto Cuaderno Venezolano de Sociología, volumen: 20, $\mathrm{N}^{\circ} 3$, pp. 501-517.

2009 «Deporte: ¿Fenómeno natural y eterno o creación socio-histórica?». En: Espacio Abierto Cuaderno Venezolano de Sociología, volumen: $18, \mathrm{~N}^{\circ} 1$.

2007 «Presente y futuro del deporte en la globalización». En: Deporte y Revolución en América Latina. Maracaibo: Ediciones del ViceRectorado Académico de la Universidad de Zulia.

AMIT, R. y P. Schoemaker

1993 «Strategic Assets and Organizational Rent». En: Strategic Management Journal, volumen: 14, pp. 33-46.

ANAND, J. y H. Singh, H.

1997 «Asset Redeployment, acquisitions and corporate strategy in declining industries». En: Strategic Management Journal, volumen: 18, pp. 99-118.

ANDREFF, Wladimir

2006 «Derechos televisivos desiguales y desequilibrio competitivo en Europa». En: La Vanguardia Dossier: El poder del fútbol, $\mathrm{N}^{\circ} 20$, pp. 30-40.

\section{APOYO CONSULTORÍA}

2004 Desarrollo e implementación de una estrategia para la mejora de la competitividad e incremento de la rentabilidad en el fútbol peruano.

ASOCIACIÓN DEPORTIVA DE FÚTBOL PROFESIONAL (ADFP)

2011 «La Asociación». En: www.adfp.org.pe. Fecha de consulta: 30/07/2011. <http://www.adfp. org.pe/portal/presentacion.php $>$.

2012 Torneo Descentralizado 2012 Copa Movistar.

BARAJAS, Ángel

2005 El valor económico del fútbol. Radiografía financiera del fútbol española. Pamplona: Eunsa.
BARNEY, J.

1991 «Firm Resources and Sustained Competitive Advantage». En: Journal of Management, volumen: 17, pp. 99-120.

BBC MUNDO

2005 Manchester United, el más rico. Jueves 17 de febrero de 2005 .

BETTIS, R.

1981 «Performance Differences in Related and Unrelated Diverified Firms». En: Strategic Management Journal, volumen: 2, pp. 379-93.

BREITBARTH, Tim y Phil Harris

2008 «The Role of Corporate Social Responsability in the Football Business: Towards the development of a conceptual model». En: European Sport Management Quarterly, volumen 8, $\mathrm{N}^{\circ}$ 2, pp. 179-206.

BLANCO, Miguel y Francisco Forcadell

2006 «El Real Madrid Club de Fútbol: La aplicación de un modelo empresarial a una entidad deportiva en España». En: Universia Business Review, Tercer trimestre 2006, pp. 36-61.

BOSCÁ, J. y V. LIERN

2008 «The Spanish Football Crisis». En: European Sport Management Quarterly, volumen: 8, $\mathrm{N}^{\circ}$ 2, pp. 165-77.

CANUT, L.

2007 «De Unicef a Bwin». En: Mundo Deportivo, volumen: $2, \mathrm{~N}^{\circ} 6, \mathrm{pp} .15$.

CASTELLS, $\mathrm{M}$.

2009 Comunicación y poder. Madrid: Alianza Editorial.

\section{CASTRO, Roberto}

2009 «Cuál es el problema con que un equipo que ascienda empieza desde la etapa distrital?». En: dechalaca.com. 28/02/2009. Fecha de consulta: 30/07/2011. <http://dechalaca.com/copaperu/ opinion/ligas-superiores-un-gran-paso $>$

Club Deportivo Universidad de San Martín de Porres (CDUSMP)

2010 Club Deportivo Universidad San Martín de Porres: 2004 - 2009. La Historia.

LIMA: MERCADOS \& NORANDINO S. A.

S.f. En: $\quad$ www.clubdeportivo.usmp.edu.pe.

Fecha de consulta: 30/07/2011. http://www. clubdeportivo.usmp.edu.pe/images/orga.jpg. 


\section{CONFEDERACIÓN SUDAMERICANA \\ DE FÚTBOL} (CONMEBOL) «Copa América 2011». En: www.conmebol. com. Fecha de consulta: 30/07/2011. < http:// www.conmebol.com/pages/estadisticas_copa_ america_2011.html>.

2007 Historial de la Copa América

s. f. a «Quienes somos - La Conmebol». En: www. conmebol.com. Fecha de consulta: 30/07/2011. $<$ http://www.conmebol.com/pages/Quienes_ Somos 2.html>

s. f. b «El Comité Ejecutivo». En: www.conmebol com. Fecha de consulta: 30/07/2011. < http:// www.conmebol.com/pages/Comite_ejecutivo. html>.

CONTRERAS, E. y L. Beneyto

2006 «Capelo da un giro a las giras del Madrid». En: Marca, volumen: 8, pp. 8.

D'ALESSANDRO, D. y M. Owens

2001 Brand Warfare: 10 Rules for Building the Killer Brand. Nueva York: McGraw Hill.

DATA SPORT: Operador Estadístico A. D. F. P.

2011 Torneo Descentralizado 2010: Fecha 15

2010a Torneo Descentralizado 2010: Liguilla: Fecha 14

2010b Torneo Descentralizado 2010: Play Off: Fecha 1

2010c Torneo Descentralizado 2010: Play Off: Fecha 2

2009a Torneo Descentralizado 2009: Liguilla: Fecha 14

2009b Torneo Descentralizado 2009: Play Off: Fecha 1

2009c Torneo Descentralizado 2009: Play Off: Fecha 2

DÁVILA, Antonio

2007 «El futuro del deporte rey». En: Revista de Antiguos Alumnos IESE Business School, volumen: abril-junio, pp. 62-6.

De Chalaca

2011 «Mercado de Pases 2011». En: dechalaca.com. 07/04/2011. Fecha de consulta: 07/08/2011. $<$ http://dechalaca.com/primera-division/ refuerzos-2011/mercado-de-pases-2011>.

2010 «Mercado de Pases 2010». En: dechalaca.com. 21/08/2010. Fecha de consulta: 07/08/2011. $<$ http://dechalaca.com/primera-division/ refuerzos-2010/mercado-de-pases-2010>.

2009 «Mercado de Pases 2009». En: dechalaca.com. 15/01/2009. Fecha de consulta: 07/08/2011. $<$ http://dechalaca.com/primera-division/ refuerzos-2009/mercado-de-pases-2009>.
DELOITTE

2012 Football Money League.

2011 Conclusiones y un análisis desde la perspectiva Latinoamericana.

DEMIL, Benoit y Xavier Lecocq

2009 «Evolución de modelos de negocio: hacia una visión de la estrategia en términos de coherencia dinámica». En: Universia Business Review, Tercer trimestre 2009, pp. 86-106.

DEPOR

2011

«La marca Perú: 58 futbolistas de nuestro país nos representan afuera». En: depor.pe. 09/05/2011. Fecha de consulta: 30/07/2011. $<$ http://depor.pe/noticia/754620/marca-peru58-futbolistas-nuestro-pais-nos-representanafuera $>$.

2010 «El peruano Josepmir Ballón ya entrena con River Plate». En: depor.pe. 22/06/2010. Fecha de consulta: 07/08/2011. <http://depor.pe/ noticia/499000/peruano-josepmir-ballon-yaentrena-river-plate>

2009 «El regreso de CNI a la Primera División ante Cristal será a estadio lleno en Iquitos». En: depor.pe. 25/02/2009. Fecha de consulta: 30/07/2011. <http://depor.pe/noticia/245748/ regreso-cni-primera-division-ante-cristalestadio-lleno-iquitos>.

DINERO

2003 «Real Madrid of Spain. Un Nuevo esquema de negocio para el deporte. ¿Funcionará?». volumen: 922 , septiembre.

DOBSON, S. y J. Goddard

2001 The Economics of Football. Cambridge: Cambridge University Press.

ECUAVISA

2010 «Deportivo Quito contrata a delantero peruano Fernández por más de US\$1.000.000 ». En: www. ecuavisa.com. Sección Deportes. 24/07/2010. Fecha de consulta: 07/08/2011. <http://www. ecuavisa.com/deporte-nacional/26375.html>.

EISENBERG, C., P. Lanfranchi, T. Mason y A. Wahl

2004 FIFA 1904-2004. Un siglo de fútbol. Madrid. Pearson Educación. Publicación oficial de la FIFA.

EISENHARDT, K.

1989 «Building Theories From Case Study Research». En: Academy of Management Review, volumen: $14, \mathrm{~N}^{\circ} 4$, pp. 532-50.

EL COMERCIO

2011a «¿Qué derecho laborales tienen los 
futbolistas profesionales?». En: elcomercio. pe. Sección Economía. 23/06/2011. Fecha de consulta: 30/07/2011. <http://elcomercio. pe/economia/802137/noticia-que-derechoslaborales-tienen-futbolistas-profesionales $>$

2011b «Plazo vence miércoles: 'U' y Alianza deben S/.131 millones a la SUNAT». En: <elcomercio. pe. Sección Deportes. 31/01/2011. Fecha de consulta: 30/07/2011. <http://elcomercio. $\mathrm{pe} / \mathrm{de}$ portes/70 6696/no ticia-plazo-vencemiercoles-u-y-alianza-deben 131 -millonessunat>

2011c «La SUNAT podría embargar los logos de la 'U' y Alianza».En:<elcomercio.pe. Sección Deportes. 09/02/2011. Fecha de consulta: 30/07/2011. $<$ http://elcomercio.pe/deportes/711267/ noticia-sunat-podria-embargar-logos-u-yalianza>

2011d «Copa América: Yoshimar Yotún, candidato a mejor jugador joven». En: <elcomercio. pe. Sección Deportes. 22/07/2011. Fecha de consulta: 07/08/2011. <http://elcomercio. pe/deportes/930941/noticia-copa-americayoshimar-yotun-candidato-peruano-mejorjugador-joven>

2010a «Encuesta de la U. de Lima: Universitario es el equipo que más hinchas tiene en la capital». En: elcomercio.pe. Sección Deportes. 25/02/2010. Fecha de consulta: 30/07/2011. $<$ http://elcomercio.pe/deportes/419568/ noticia-encuesta-lima-alianza-universitarioson-candidatos-llevarse-titulo>

2010b «Gana con la camiseta: Alianza Lima es el club peruano que más recibe por sponsors». En: elcomercio.pe. Sección Deportes. 45/02/2010. Fecha de consulta: 07/08/2011. <http:// elcomercio.pe/deportes/419023/noticia-ganacamiseta-alianza-lima-club-peruano-que-masrecibe-sponsors $>$

104

\section{EL MUNDO}

2011 «El éxito integral de Uruguay». En: www. elmundo.es. 20/07/2011. Fecha de consulta: 30 de noviembre de 2011. <http://www.elmundo. es/america/2011/07/20/deportes/1311150894. html>

EL PAÍS

2011 «El proyecto Forlán». En: www.elpais.cr. 10/08/2011. Fecha de consulta: 10/08/2011. $<$ http://www.elpais.cr/articulos.php?id=50892>

2003 «La explotación de la marca Real Madrid supone ya el $35 \%$ de los ingresos del club», 17 de octubre.

2002 «Ronaldo, el mejor para hacer caja. El tirón del campeón mundial permite al Madrid abrirse paso en el fabuloso mercado japonés», $3 \mathrm{de}$ diciembre.
2001 «El Madrid recompra la explotación de sus palcos y prevé ganas 12000 millones en 8 años», 18 de agosto.

2001 «El Madrid quiere golear con la marca», 15 de noviembre.

2000 «El sanzismo es lo de Figo pero todos los días». Entrevista a Florentino Pérez, 13 de julio de 2000.

\section{EMPRENDEDORES}

2001 «La gestión de lo imprevisible. Técnicas de Empresa aplicadas a un club deportivo». Número 50. Noviembre

2000 «Merienda de blancos. Las claves de la crisis del Real Madrid». Número 29. Febrero de 2000.

\section{ENFOQUE ECONÓMICO}

2010 Enfoque económico: revista de negocios en el Perú, año $\mathrm{V}, \mathrm{n}^{\circ} 72$.

\section{FC BARCELONA}

2009 Memoria anual, Barcelona.

2008 Memoria anual, Barcelona.

2008 Memoria anual, Barcelona.

2007 Memoria anual, Barcelona.

2006 Memoria anual, Barcelona.

2005 Memoria anual, Barcelona.

2004 Memoria anual, Barcelona.

2003 Memoria anual, Barcelona.

\section{FC BUSINESS}

2008 «El Barca, líder absolut en popularitat». $\mathrm{N}^{\circ} 2$, pp. 1.

\section{FEDERACIÓN PERUANA DE FÚTBOL (FPF)}

s. f. «Historia». En: www.fpf.com. Fecha de consulta: 30/07/2011. <http://www.fpf.com. $\mathrm{pe} />$

FEDERATION INTERNATIONALE DE FOOTBALL ASSOCIATION (FIFA)

2011a «Copa Mundial de la FIFA». En: es.fifa.com. Fecha de consulta: 30/07/2011.<http://es.fifa. com/worldcup/archive/index.html $>$

2011 b «Partidos». En: es.fifa.com. Fecha de consulta: 30/p7/2011. <http://es.fifa.com/u17worldcup/ matches/index.html>

s. f. a «La FIFA». En: es.fifa.com. Fecha de consulta: 30/07/2011. <http://es.fifa.com/aboutfifa/ organisation/index.html>

s. f. b «Confederaciones». En: es.fifa.com. Fecha de consulta: 30/07/2011. <http://es.fifa.com/ aboutfifa/organisation/confederations/index. html>

s. f. c «Clasificación mundial». En: es.fifa.com. Fecha de consulta: 30/07/2011. <http://es.fifa.com/ worldfootball/ranking/lastranking/gender $=\mathrm{m} /$ fullranking.html> 
FORCADELL, F.

2006 «Crecimiento empresarial, estrategia corporativa y recursos ». En: Academy of Management Review, volumen: 14, $\mathrm{N}^{\circ} 4$, pp. 532-50.

FORSTER, J. y N. Pope

2004 The political economy of global sporting organisations. Londres: Routledge.

GASTAÑADUY, Alfonso

2003 «Marketing de Instituciones Deportivas: de las relaciones públicas a la valoración de la imagen corporativa como activo intangible». En: Esan - Cuadernos de Difusión, Año 8, $\mathrm{N}^{\circ} 14$, pp. 4161.

\section{GESTIÓN}

2011a «Alianza, U y Boys pagarán a la SUNAT para evitar embargos». En: gestion.pe. Sección Negocios. 21/03/2011. Fecha de consulta: 30/07/2012. <http://gestion.pe/noticia/730796/ alianza-boys-pagaran-sunat-evitar-embargos $>$

2011 b «n el Perú, dos futbolistas peruanos ganan 25 mil dólares mensuales». En gestión.pe. 06/12/2011. Fecha de Consulta: 30/07/2012.

2010 «Gobierno publica ley que promueve transformación de clubes en S.A.». En: gestion. pe. Sección Economía. 31/01/2010. Fecha de consulta: 30/07/2011. <http://gestion.pe/ noticia/730796/alianza-boys-pagaran-sunatevitar-embargos>

GINESTA, Xavier

2011 «El fútbol y el negocio del entretenimiento global. Los clubes como multinacionales del ocio». En: Comunicación y Sociedad, volumen: 24, $\mathrm{N}^{\circ} 1$, pp. 141-66.

GQ LATINOAMÉRICA

2011 «Corazón limeño». En: GQ Latinoamérica. Julio de 2011. Pp. 120-25.

GREEN, M.

2006 «From 'sport for all' to not about 'sport' at all? Interrogating sport policy interventions in the United Kingdom». En: European Sport Management Quarterly, volumen 6, $\mathrm{N}^{\circ} 3$, pp. 217-38.

HAMIL, S., J. Michie, Ch. Oughton y S. Warby

2000 Football in the digital age. Edimburgo: Mainstream Publishing.

HEEREN, Carlos

2010 «El negocio del fútbol». En: Enfoque económico: revista de negocios en el Perú, año $\mathrm{V}, \mathrm{n}^{\mathrm{0}} 72$, p. 7. s. f. «El negocio del fútbol en el Perú y la búsqueda de utilidades». En: Punto de Equilibrio. Fecha de consulta: 29/07/2011. <http:// www.pun todeequilibrio.com.pe/punto_ equilibrio/01i.php?pantalla $=$ noticia\&id $=15401$ \&bolnum_key=17\&serv_key=2100>

HUAYTALLA, Ricardo

2010 Entrevista realizada a Ricardo Huaytalla, Jefe de prensa del Club deportivo de la Universidad de San Martín de Porres. Junio de 2010.

\section{INDEX MUNDI}

2011 «Uruguay población». En: www.indexmundi. com. Fecha de consulta: 30/07/2011. <http:// www.indexmundi.com/es/uruguay/poblacion. html>.

INFOS

2012 «El silencio de los insolventes». En: Infos. Fecha de consulta: 30/07/2012. <http://www. infos.pe/2012/03/futbol-el-silencio-de-losinsolventes/>.

\section{INSTITUTO NACIONAL DE ESTADÍSTICA E INFORMÁTICA} (INEI)

s. f. «Perú: Población total, censada y tasa de omisión, según censos realizados, 1940 - 2007». En: INEI. Fecha de consulta: 30/07/2011. <http:// www.inei.gob.pe/perucifrasHTM/inf-dem/ cuadro.asp?cod=11224\&name=po12\&ext=gif.

INSTITUTO PERUANO DEL DEPORTE (IPD)

s. f. «Qué es el IPD». En: www.ipd.gob.pe. Fecha de consulta: 30/07/2011. < http://www.ipd.gob.pe/ index.php/que-es-el-ipd $>$.

INTERNATIONAL FEDERATION OF FOOTBALL HISTORY AND STATISTICS (IFFHS)

2011 «Las Ligas más Fuertes del Mundo en 2011: Primeras tendencias». En: www.iffhs.de. Fecha de consulta: 30/07/2011.< http://www.iffhs.de/ ?b6e36fa3002f70d00ee2d17f7370eff3702bb0a $35 \mathrm{~b} 10>$.

2010 «La Mejor Liga de Fútbol del Mundo 2010». En: www.iffhs.de. Fecha de consulta: 30/07/2011.< http://www.iffhs.de/?b6e28fa3002f76b55a769 17f7370eff3702bb0a35b12>.

2009 «La Mejor Liga de Fútbol del Mundo 2009». En: www.iffhs.de. Fecha de consulta: 30/07/2011.< http://www.iffhs.de/?b6e28fa3002f76b55a66 017f7370eff3702bb0a35b17http://es.fifa.com/ aboutfifa/organisation/index.html>.

S. f. «La historia de la IFFHS». En: www.iffhs.de. Fecha de consulta: 30/07/2011. <http://www. iffhs.de/?b3136fe3016e23617ff3c17f7370eff37 02bb0a35b07>. 
JENNINGS, A.

1996 Los nuevos señores de los anillos. Barcelona: Ediciones de la Tempestad.

KASE, $\mathrm{K}$

2006 Real Madrid CF-FC Barcelona: Análisis de las estrategias económica y deportiva del periodo 2000-2006. En: IESE. Fecha de consulta: 12 de abril de 2010. En: http://www.iese.edu/ research/pdfs/OP-06-12.pdf.

\section{LA REPÚBLICA}

2008 «FIFA levantó sanción a Federación Peruana de Fútbol». En: www.larepublica.pe. 20/12/2008. Fecha de consulta: 30/07/2011. <http://www. larepublica.pe/20-12-2008/fifa-levantosancion-federacion-peruana-de-futbol $>$.

LEE, F.

2005 «Spectacle and Fandom: Media Discursee in Two Soccer Events in Hong Kong». En: Sociology of Sport Journal, $\mathrm{N}^{\circ} 22$, pp. 194-213.

LÍBERO

2011a «Sepa algo más del traspaso de Rinaldo Cruzado al Chievo Verona». En: www.libero. pe. 12/17/2011. Fecha de consulta: 07/08/2011. <http://www.libero.pe/sepa-algo-mas-deltraspaso-de-rinaldo-cruzado-al-chievo-verona2011-07-12>.

2011 b "Cachito” Ramírez será suplente de lujo en el Corinthians». En: www.libero.pe. 14/01/2011. Fecha de consulta: 07/08/2011. <http://www. libero.pe/cachito-ramirez-sera-suplente-delujo-en-el-corinthians-2011-01-14>.

2011c «Fútbol peruano: Juvenal Silva agarró dinero de Cienciano presupuestado para el 2015 ». En: www.libero.pe. 18/02/2011. Fecha de consulta: 07/08/2011. <http://www.libero.pe/ futbol-peruano-juvenal-silva-agarro-dinerode-cienciano-presupuestado-para-el-20152011-02-18>.

2010a «La santa Niza». En: www.libero.pe. 15/11/2010. Fecha de consulta: 07/08/2011.<http://www. libero.pe/la-santa-niza-2010-11-15>.

2010 b «rensa de Argentina: Giancarlo Carmona jugará por San Lorenzo». En: www.libero.pe. 22/12/2010. Fecha de consulta: 07/08/2011. $<$ http://www.libero.pe/prensa-de-argentinagiancarlo-carmona-jugara-por-san-Iorenzo2010-12-22>.

LIGA DE FÚTBOL PROFESIONAL

2004 Impacto del Fútbol Profesional en la Economía Española

LUCIFORA, C. ; SIMMONS, R.

2003 «Superstars Effects in Sport: Evidence from
Italian Soccer». En: Journal of Sports Economics, volumen: $4, \mathrm{~N}^{\circ} 1$, pp. 35-55.

MAGUIRE, J.

1999 Global Sport. Cambridge: Polity Press.

MARILEES, B., D. Ges y D. O'Brien

2005 «Marketing Stakeholder Analysis Branding the Brisbane Goodwill Games».En: European Journal of Marketing, volumen: 39, pp. 1060-77.

MASON, D.

1999 «What is the sports product and who buys it? The marketing of professional sports leagues». En: European Journal of Marketing, volumen 33.

\section{NETJOVEN}

2010a «Roberto Guizazola es el nuevo refuerzo de Rosario Central». En: www.netjoven.pe. 20/07/2010. Fecha de consulta: 07/08/2011. <http://www.netjoven.pe/noticias/45133/ Roberto-Guizasola-es-el-nuevo-refuerzo-deRosario-Central.html>

2010b «Fútbol Peruano: Pagarían 4 Millones a Universitario de Deportes por trasmisión de partidos». En: www.netjoven.pe. 02/12/2010. Fecha de consulta: 07/08/2011. <http://www. netjoven.pe/noticias/52034/Futbol-PeruanoPagarian-4-Millones-a-Universitario-deDeportes-por-trasmision-de-partidos.html>

OUGHTON, C.

2004 «Risk, governance and regultatory issues facing professional football». En: World Sports Law Report, volumen 2, $\mathrm{N}^{\circ} 8$, pp. 18-21.

OUGHTON, C. ; MILLS, C. ; MC CLEAN, M. ; HUNT, P.

2003 Back home: Returning football clubs to their communities. Londres: Mutuo.

PÁGINA 12

2011 «El librito del Maestro». En www.pagina12. com.ar. 25/07/2011. Fecha de c o n s u 1 t a : 10/08/2011. <http://www.pagina12.com.ar/ diario/suplementos/libero/subnotas/61373228-2011-07-27.html>

PEREZ, F.

2001 Discurso ante la asamblea de socios compromisarios del Real Madrid, 23 de Septiembre.

\section{PERÚ CAMPEÓN}

s.f. «Para el Perú que lo mira por TV». Fecha de consulta: 07/08/2011. < http://www. perucampeon.com/seleccion-peruana/para-elperu-que-lo-mira-por-tv.html> 
PERÚ 21

2011

«La 'Culebra' ya es del Sporting de Lisboa». En: peru21.pe. Sección Deportes. 30/04/2011. Fecha de consulta: 07/08/2011. <http://peru21. pe/noticia/750397/culebra-ya-sporting-lisboa $>$

2010 «WIlmer Aguirre firmó por San Luis de Potosí». En: peru21.pe. Sección Deportes. 22/05/2010. Fecha de consulta: 07/08/2011. <http://peru21. pe/noticia/482412/wilmer-aguirre-firmo-sanluis>

PIZARRO, Luis

2010 «El negocio del fútbol». En: Enfoque económico: revista de negocios en el Perú, año $\mathrm{V}, \mathrm{n}^{\circ} 72$, pp. 15-6.

REAL MADRID

2003 Informe Anual Abreviado 2002-2003

2003 Boletín de Socios. Número 11. Noviembre diciembre.

2001 Informe Anual Abreviado 2000-2001.

RICE-OXLEY, M.

2003 European Soccer trips over bad economics. En: The Christian Science Monitor. Junio de 2003.

ROSADO, Roberto

2011 «El negocio del fútbol en Perú movió US\$ 30 millones en el 2010». En: Gestión [Lima]. 03/03/2011. Sección Negocios.

SAMARANCH, Juan.

2002 Juan Antonio Samaranch: Memorias Olímpicas. Barcelona: Editorial Planeta.

SANDY, R., P. Sloane y M. Rosentraub

2004 The economics of football - an international perspective. Londres: PalgraveMacmillan.

SORIANO, Ferran

2009 La pilota no entra per atzar, Ara llibres, Badalona,
SUDGEN, J.

2002 Scum Airways - inside football's underground economy. Edimburgo: Mainstream.

SUPERINTENDENCIA NACIONAL DE ADMINISTRACIÓN TRIBUTARIA (SUNAT)

s. f. «¿Qué es la SUNAT?». En: www.sunat.gob.pe. Fecha de consulta: 30/07/2011. <http://www. sunat.gob.pe/institucional/quienessomos/ index.html>

SUPERINTENDENCIA NACIONAL DE REGISTROS PÚBLICOS (SUNARP)

s. f. «Sociedad anónima». En: www.sunarp.gob.pe. Fecha de consulta: 30/07/2011. <http://www. sunarp.gob.pe/Aten24h/pdf/Anexo02.pdf>

\section{SZYIMANSKY, S}

1998 «Why is Manchester United so successful?». En: Business Strategy Review, volumen: 9, $\mathrm{N}^{\circ} 4$, pp. 47-54.

\section{THE OBSERVER}

2002 «Real Madrid: the best team ever?» Septiembre.

VILLEGAS, Miguel y Elkin SOTELO

2007 «Woodman dice que Burga debe acatar el fallo y dar un paso al costado». En: El Comercio [Lima]. 24/03/2007. Sección Tema del día.

WILLIAMS, J.

1994 «The Local and the Global in English Soccer and the Rise of Satellite Television». En: Sociology of Sports Journal, volumen 11, pp. 376-97. 